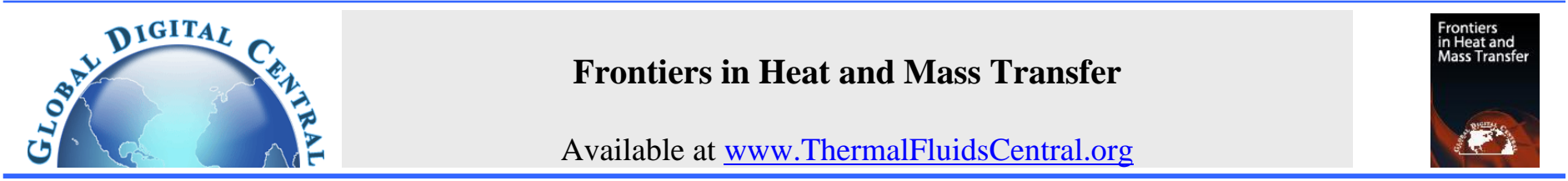

\title{
A LARGE PARAMETER SPECTRAL PERTURBATION METHOD FOR NONLINEAR SYSTEMS OF PARTIAL DIFFERENTIAL EQUATIONS THAT MODELS BOUNDARY LAYER FLOW PROBLEMS
}

\author{
T. M. Agbaje ${ }^{\mathrm{a}, \mathrm{b}}$, S. S. Motsa ${ }^{\mathrm{a},}{ }^{*}$, S. Mondal ${ }^{\mathrm{c}, \dagger}$, P. Sibanda ${ }^{\mathrm{a}}$ \\ ${ }^{a}$ School of Mathematics,Statistics and Computer Sciences, University of KwaZulu-Natal, Pietermaritzburg, Private Bag X01 Scottsville-3209, South \\ Africa \\ ${ }^{\mathrm{b}}$ DST-NRF Centre of Excellence in Mathematical and Statistical Sciences (CoE-MaSS), Private Bag 3, Wits 2050, Johannesburg, South Africa \\ ${ }^{\mathrm{c}}$ Department of Mathematics, Amity University, Kolkata, Newtown- 700135, West Bengal, India
}

\begin{abstract}
In this work, we present a compliment of the spectral perturbation method (SPM) for solving nonlinear partial differential equations (PDEs) with applications in fluid flow problems. The (SPM) is a series expansion based approach that uses the Chebyshev spectral collocation method to solve the governing sequence of differential equation generated by the perturbation series approximation. Previously the SPM had the limitation of being used to solve problems with small parameters only. This current investigation seeks to improve the performance of the SPM by doing the series expansion about a large parameter. The new method namely the large parameter spectral perturbation method (LSPM) combines the idea of asymptotic analysis approach with numerical solution techniques. In the (LSPM), the resulting equations from the asymptotic expansion are solved numerically using the Chebyshev spectral method. The purpose of this study is to extend the existing spectral perturbation method (SPM) which was used for small parameters to be suitable for problems with large parameters. The applicability of the (LSPM), is tested on systems of earlier reported nonlinear partial differential equations that describe boundary layer fluid flow problems. The validity of the (LSPM) numerical solutions is verified by comparing with published results and the bivariate Chebyshev spectral quasilinearisation method (BSQLM) and an excellent agreement were observed. The (BSQLM) is a numerical method that blends the quasilinearisation method, the Chebyshev spectral method, and the bivariate Lagrange interpolation method. One of the advantages of this approach is that it gives results in a fraction of seconds. We remark also that simple decoupled linear systems formulas are derived for generating the solutions in the form of decoupled linear systems. Tables are generated to present error and convergence properties of the LSPM.
\end{abstract}

Keywords: Spectral Perturbation Method; Bivariate Chebyshev spectral quasilinearisation method; Chebyshev spectral collocation method.

\section{INTRODUCTION}

Nonlinear partial differential equations (PDEs) arise in various fields of science and engineering problems such as mathematical modelling, heat transfer, fluid mechanics, quantum mechanics and many applications in biological process. In addition, there is a large volume of published literature describing the numerous important of engineering and science applications of boundary layer flows. Examples include the cooling and drying of paper and textiles, aerodynamic extrusion of plastic sheets, cooling of metallic plates in a cooling bath. The equations defined by systems of nonlinear partial differential equations (PDEs) are often difficult to solve exactly. As a result of the difficulties often encountered in solving these nonlinear partial differential equations exactly, some researchers have de- veloped both numerical and analytical method for finding analytical and approximate solutions to these complicated nonlinear partial differential equations. These numerical and analytical methods have their own advantages and limitations. Hence, there is need to improve the performance of these existing numerical and analytical methods.

An innumerable amount of literature now exists on asymptotic solution method of boundary layer fluid flow problems modelled by nonlinear PDEs. These includes the study of Hossain and Paul (2001a,b), who investigated the flow of a free convection from a vertical permeable circular cone with non-uniform surface temperature and surface heat flux respectively, Chamkha et al. (2003) who studied the unsteady MHD rotating flow over a rotating sphere near the equator, Takhar et al. (2003) who considered the unsteady three-dimensional MHD-boundary-layer flow due to

\footnotetext{
${ }^{*}$ University of Swaziland, Private Bag 4, Kwaluseni, Matsapha M201, Swaziland

${ }^{\dagger}$ Corresponding author.Email: sabya.mondal.2007@gmail.com
} 
the impulsive motion of a stretching surface equations and employed the asymptotic method to obtain asymptotic solutions for large values of the independent variable. Free convection in micropolar fluids over a uniformly heated vertical plate was investigated by Gorla et al. (1998) and asymptotic solutions were presented for distances away from the leading edge. Bhattacharyya (2013) examined boundary layer stagnationpoint flow of Casson fluid and heat transfer towards a shrinking/stretching sheet is studied. Recently, Oyelakin et al. (2017) studied the effects of thermal radiation, heat and mass transfer on the unsteady magnetohydrodynamic(MHD) flow of a three dimensional Casson nanofluid using the spectral quasi-linearisation method. The partial differential equations governing the unsteady MHD flow on a rotating cone in a rotating fluid were solved by Roy et al. (1998), asymptotic solutions were presented for large magnetic parameter $M$. Natural convection flow from a vertical permeable flat plate with variable surface temperature and species concentration was studied by Hussain et al. (2000) using the perturbation method, asymptotic method, local non-similarity method and Kellerbox method. Saha et al. Saha et al. (2007) used the regular perturbation method, asymptotic method, implicit finite difference method together with Keller-box scheme, and the local non-similarity method to solve the effect of Hall current on the MHD laminar natural convection flow from a vertical permeable flat plate with uniform surface temperature. Slaouti et al. (2002) solved the equations modelling the spin-up and spin-down of a viscous fluid over a heated disk rotating in a vertical plane in the presence of a magnetic field and a buoyancy force and also developed an asymptotic analysis for a large magnetic parameter. An asymptotic solution for large values of the independent variable $\eta$ was generated by Takhar et al. (2001) who investigated the unsteady MHD-boundary-layer of a source and vortex flow adjacent to a stationary surface and obtained a closed form solution for large values of the suction parameter. The abovementioned authors solved the resulting asymptotic perturbation equations analytically and their results only yield the first, second and a maximum of a third-order asymptotic solution. The problem associated with the analytical approach used by all the above-mentioned authors in solving the resulting asymptotic perturbation equations is that higher order asymptotic perturbation equations may be impossible to solve exactly beyond a maximum of third-order approximation. This is because it takes far too long to generate higher order solution even with the use of a computer.

This current investigation serves to first present a Chebyshev spectral based approach that addresses the problem encountered by previous researchers on this subject in attempting to solve higher order asymptotic perturbation equations exactly. The method namely the large parameter spectral perturbation method (LSPM) is a method which blends the idea from asymptotic analysis with numerical solution techniques. The Chebyshev spectral method is used to obtain a numerical approximate solution of the higher order asymptotic perturbation approximations which may be impossible to solve exactly. We demonstrate that using the Chebyshev spectral method to integrate the higher order asymptotic perturbation equations gives very accurate numerical results even for complicated nonlinear partial differential equations in a computationally fast manner. The Chebyshev spectral was chosen in this work because spectral methods are well documented for their high level of accuracy and also with the spectral methods only few grid points are required to obtain accurate results. In addition, we show that using the spectral methods leads to a significant saving in computational time. This is in contrast with the analytical approach earlier employed by previous researchers because, in the analytical approach, it takes too long to solve higher order asymptotic equations even with the use of a scientific software but with the spectral methods, higher order asymptotic solutions are generated in a fraction of seconds. Secondly, in this present study, we aim to introduce the (LSPM) as a compliment of the existing spectral perturbation method (SPM) which was used for small parameters and its limited in its application. The (SPM) is limited in the sense that the (SPM) is valid for small parameters and the (LSPM) approach is valid for large parameters. The (SPM) is a series expansion based method that extends the use of the traditional perturbation scheme coupled with the Chebyshev spectral method. The (SPM) was used in the study carried out by Agbaje and Motsa (2015) to address some of the drawbacks of the traditional perturbation method and the approach was able to correct some of the conclusions drawn by researchers regarding perturbation methods. It was observed in the study conducted by Agbaje and Motsa (2015) on the SPM that the approach though accurate is not suitable for large parameters. As a result of that observation made on the (SPM), we aim to extend the range of validity of the (SPM) by expanding about a large physical parameter so as to make the usual (SPM) robust and extends its application to new areas. Also, we show that the convergence speed of the usual (SPM) can be improved even when $\xi$ is large by doing the series expansion about a large physical parameter. Furthermore, simple decoupled linear systems formulas for generating the solutions in the form of decoupled linear systems were derived in this study. This is an advantage of this method because the derived simple linear systems formulas enable us to solve the equations independently of each other. In order to demonstrate the applicability of the (LSPM), we consider three earlier reported boundary layer flow problem modelled by coupled systems of two and three nonlinear PDEs. The accuracy of the (LSPM) numerical results was compared with the bivariate quasilinearisation method (BSQLM) and the published results of Saha et al. (2007) and Hossain and Paul (2001a,b) and a good agreement was observed.

\section{GOVERNING SYSTEMS OF NONLINEAR PARTIAL DIFFERENTIAL EQUATIONS}

In this section, we present the systems of nonlinear partial differential equations (PDEs) that describes the different types of boundary layer flows that will be investigated in this study. In order to demonstrate the applicability of the (LSPM) numerical method of solution, we consider three various types of nonlinear PDEs.

\subsection{Effect of Hall current on the MHD laminar natural convection flow from a vertical permeable flat plate with uniform surface temperature}

We consider the effect of hall current on the MHD laminar natural convection flow from a vertical permeable flat plate with uniform surface temperature investigated by Saha et al. Saha et al. (2007). The governing nonlinear partial differential equations are given in Saha et al. (2007) in dimensionless form as:

$f^{\prime \prime \prime}+\frac{3}{4} f f^{\prime \prime}-\frac{1}{2} f^{\prime 2}+\xi f^{\prime \prime}+\theta-\frac{M}{\left(1+m^{2}\right)}\left(f^{\prime}+m g\right)=\frac{1}{4} \xi\left[f^{\prime} \frac{\partial f^{\prime}}{\partial \xi}-f^{\prime \prime} \frac{\partial f}{\partial \xi}\right]$

$g^{\prime \prime}+\frac{3}{4} f g^{\prime}-\frac{1}{2} f^{\prime} g+\xi g^{\prime}-\frac{M}{\left(1+m^{2}\right)}\left(g-m f^{\prime}\right)=\frac{1}{4} \xi\left[f^{\prime} \frac{\partial g}{\partial \xi}-g^{\prime} \frac{\partial f}{\partial \xi}\right]$,

$\frac{1}{P r} \theta^{\prime \prime}+\frac{3}{4} f \theta^{\prime}+\xi \theta^{\prime}=\frac{1}{4} \xi\left[f^{\prime} \frac{\partial \theta^{\prime}}{\partial \xi}-\theta^{\prime} \frac{\partial f}{\partial \xi}\right]$

In the above equation, $M$ is the magnetic field, $\xi$ is the transpiration parameter, $m$ is the hall parameter, $(\operatorname{Pr}=v / \alpha)$ is the Prandtl number and prime denotes differentiation of the functions with respect to $\eta$. The corresponding boundary conditions to the above equations are:

$$
\begin{aligned}
& f(\xi, 0)=f^{\prime}(\xi, 0)=0, \quad g(\xi, 0)=0, \quad \theta(\xi, 0)=1 \\
& f^{\prime}(\xi, \infty)=g(\xi, \infty)=\theta(\xi, \infty)=0 .
\end{aligned}
$$

The physical quantities of interest are the skin-friction and Nusselt number, which may be calculated from the following expressions and are given in Saha et al. (2007) as:

$$
C_{f x} G r_{x}^{-3 / 4}=f^{\prime \prime}(\xi, 0), \quad N u_{x} G r_{x}^{-1 / 4}=-\theta^{\prime}(\xi, 0) .
$$




\subsection{Free convection from a vertical permeable circular cone with non-uniform surface temperature}

We investigate a steady two-dimensional laminar free convection flow past a non-isothermal vertical porous cone with variable surface temperature. The governing non-similarity system of partial differential equations are expressed in dimensionless form as Hossain and Paul (2001a):

$$
\begin{aligned}
& f^{\prime \prime \prime}+\frac{n+7}{4} f f^{\prime \prime}-\frac{n+1}{2} f^{\prime 2}+\theta+\xi f^{\prime \prime}=\frac{1-n}{4} \xi\left(f^{\prime} \frac{\partial f^{\prime}}{\partial \xi}-f^{\prime \prime} \frac{\partial f}{\partial \xi}\right), \\
& \frac{1}{P r} \theta^{\prime \prime}+\frac{n+7}{4} f \theta^{\prime}-n f^{\prime} \theta+\xi \theta^{\prime}=\frac{1-n}{4} \xi\left(f^{\prime} \frac{\partial \theta}{\partial \xi}-\theta^{\prime} \frac{\partial f}{\partial \xi}\right),
\end{aligned}
$$

where $\operatorname{Pr}=\nu / \alpha$ is the Prandtl number, $\xi$ is the dimensionless suction parameter. The appropriate corresponding boundary conditions are;

$$
\begin{aligned}
& f(\xi, 0)=0, \quad f^{\prime}(\xi, 0)=0, \quad f^{\prime}(\xi, \infty)=0, \\
& \theta(\xi, 0)=1, \quad \theta(\xi, \infty)=0 .
\end{aligned}
$$

The skin friction coefficient $C_{f x}$ and the Nusselt number $N u_{x}$ describe the shear-stress and heat flux rate at the surface, respectively, and are defined by Hossain and Paul (2001a) as:

$$
\begin{aligned}
& C_{f x} G r_{x}^{1 / 4}=f^{\prime \prime}(\xi, 0), \\
& \frac{N u_{x}}{G r_{x}^{1 / 4}}=-\theta^{\prime}(\xi, 0) .
\end{aligned}
$$

\subsection{Free convection from a vertical permeable circular cone with non-uniform surface heat flux}

We consider a steady two-dimensional laminar free convection flow of a viscous incompressible fluid with temperature, $T$, from a vertical porous cone with variable heat flux. The governing systems of nonlinear partial differential equations are given in Hossain and Paul (2001b) in dimensionless form as:

$$
\begin{gathered}
f^{\prime \prime \prime}+\frac{m+9}{5} f f^{\prime \prime}-\frac{2 m+3}{2} f^{\prime 2}+\phi+\xi f^{\prime \prime}=\frac{1-m}{5} \xi\left(f^{\prime} \frac{\partial f^{\prime}}{\partial \xi}-f^{\prime \prime} \frac{\partial f}{\partial \xi}\right), \\
\frac{1}{P r} \phi^{\prime \prime}+\frac{m+9}{5} f \phi^{\prime}-\frac{4 m+1}{5} f^{\prime} \phi+\xi \phi^{\prime}=\frac{1-m}{5} \xi\left(f^{\prime} \frac{\partial \phi}{\partial \xi}-\phi^{\prime} \frac{\partial f}{\partial \xi}\right)
\end{gathered}
$$

where $\operatorname{Pr}=\nu / \alpha$ is the Prandtl number. The corresponding boundary conditions are Hossain and Paul (2001b);

$$
\begin{aligned}
& f(\xi, 0)=0, \quad f^{\prime}(\xi, 0)=0, \quad f^{\prime}(\xi, \infty)=0, \\
& \phi^{\prime}(\xi, 0)=-1, \quad \phi(\xi, \infty)=0 .
\end{aligned}
$$

The skin friction coefficient $C_{f x}$ and the Nusselt number $N u_{x}$ describe the shear-stress and heat flux rate at the surface, respectively, and are defined by Hossain and Paul (2001b) as:

$$
\begin{aligned}
& \frac{1}{2} C_{f x} G r_{x}^{1 / 5}=f^{\prime \prime}(\xi, 0), \\
& \frac{N u_{x}}{G r_{x}^{1 / 5}}=\frac{1}{\phi(\xi, 0)}
\end{aligned}
$$

\section{METHOD OF SOLUTION}

In this section, we present the large parameter spectral perturbation method (LSPM) solution for the problems described in the previous section. Asymptotic perturbation analysis is carried out on the set of partial differential equations described in the previous section and the resulting differential equations are solved using the Chebyshev spectral method. Perturbation methods, in general, construct a solution for a problem by generating series expansions of the perturbation parameter. In perturbation methods, higher order perturbation approximations are difficult to get which may result in less accurate results if only one or two series solutions are used. For instance, Hossain and Paul (2001a,b) presented the first order asymptotic approximate solutions for large perturbation parameter using the analytical approach. Hossain and Paul (2001a,b) analytical approach yielded only the first order approximate asymptotic solution because higher order solutions may be difficult to obtain exactly even with the use of a computer. Below, the development of the large parameter perturbation approach and the application of the Chebyshev spectral method to solve the resulting ordinary differential equations is being discussed.

\subsection{Large Parameter Spectral Perturbation Method Solution for large $(\xi)$ for Equations (1- 3)}

We shall give attention to the solutions of equations $(1-3)$ when $\xi$ is large. The order of magnitude of different terms in $(1-3)$ shows that the largest in (1) are $f^{\prime \prime \prime}$ and $\xi f^{\prime \prime}$, in (2) $g^{\prime \prime}$ and $\xi g^{\prime}$, and $\theta^{\prime \prime}$ and $\xi \theta^{\prime}$ in (3). Both the terms have to be balanced in the respective equations and the only way to do this is to assume that $\eta$ to be small and its derivatives are large. Given that $\theta=O(1)$ as $\xi \rightarrow \infty$, it is necessary to find appropriate scaling for $f$ and $\eta$. On balancing the $f^{\prime \prime \prime}, \theta$ and $\xi f^{\prime \prime}$ terms in (1), it is found that $\eta=O\left(\xi^{-1}\right)$ and $f=O\left(\xi^{-3}\right)$ as $\xi \rightarrow \infty$. Therefore, the following transformations are introduced to switch the equations for small $\xi$ to those for large $\xi$;

$$
f=\xi^{-3} F(\xi, \bar{\eta}), \quad \bar{\eta}=\xi \eta, \quad g=\xi^{-2} G(\xi, \bar{\eta}), \quad \theta=\Theta(\xi, \bar{\eta}) .
$$

Substituting these transformations given in (14) into equations (1 - 3), we obtain the following equations:

$$
\begin{aligned}
& F^{\prime \prime \prime}+F^{\prime \prime}+\Theta-\frac{M}{\left(1+m^{2}\right)} \xi^{-2}\left(F^{\prime}+m G\right)=\frac{1}{4} \xi^{-3}\left[F^{\prime} \frac{\partial F^{\prime}}{\partial \xi}-F^{\prime \prime} \frac{\partial F}{\partial \xi}\right] \\
& G^{\prime \prime}+G^{\prime}-\frac{M}{\left(1+m^{2}\right)} \xi^{-2}\left(G-m F^{\prime}\right)=\frac{1}{4} \xi^{-3}\left[F^{\prime} \frac{\partial G}{\partial \xi}-G^{\prime} \frac{\partial F}{\partial \xi}\right] \\
& \Theta^{\prime \prime}+\operatorname{Pr} \Theta^{\prime}=\frac{1}{4} \operatorname{Pr} \xi^{-3}\left[F^{\prime} \frac{\partial \Theta}{\partial \xi}-\Theta^{\prime} \frac{\partial F}{\partial \xi}\right] .
\end{aligned}
$$

The corresponding boundary conditions are given as ;

$$
\begin{aligned}
& F(\xi, 0)=F^{\prime}(\xi, 0)=0, \quad G(\xi, 0)=0, \quad \Theta(\xi, 0)=1, \\
& F^{\prime}(\xi, \infty)=G(\xi, \infty)=\Theta(\xi, \infty)=0 .
\end{aligned}
$$

Since $\xi$ is large, solutions of equations (15 - 17) is obtained using the perturbation method. Hence, we expand the functions $F(\bar{\eta}), G(\bar{\eta})$ and $\Theta(\bar{\eta})$ in powers of $\xi^{-2}$ as given below;

$$
\begin{aligned}
& F(\xi, \bar{\eta})=\sum_{k=0}^{\infty} \xi^{-2 k} F_{k}(\bar{\eta}), \\
& G(\xi, \bar{\eta})=\sum_{k=0}^{\infty} \xi^{-2 k} G_{k}(\bar{\eta}), \\
& \Theta(\xi, \bar{\eta})=\sum_{k=0}^{\infty} \xi^{-2 k} \Theta_{k}(\bar{\eta}) .
\end{aligned}
$$

Substituting equations (19 - 21) into equations (15) - (17) and then equating the coefficients of like powers of $\xi$, we obtain the equations for $k=0$ as;

$$
\begin{aligned}
& F_{0}^{\prime \prime \prime}+F_{0}^{\prime \prime}+\Theta_{0}=0, \\
& G_{0}^{\prime \prime}+G_{0}^{\prime}=0, \\
& \Theta_{0}^{\prime \prime}+\operatorname{Pr} \Theta_{0}^{\prime}=0,
\end{aligned}
$$

subject to the following boundary conditions

$$
\begin{aligned}
& F_{0}(0)=F_{0}^{\prime}(0)=0, \quad G_{0}(0)=0, \quad \Theta_{0}(0)=1, \\
& F_{0}^{\prime}(\infty)=G_{0}(\infty)=\Theta_{0}(\infty)=0 .
\end{aligned}
$$

The equations for $k=1$, corresponding to order $O\left(\xi^{-2}\right)$ are given as;

$$
\begin{aligned}
& F_{1}^{\prime \prime \prime}+F_{1}^{\prime \prime}=\frac{M}{\left(1+m^{2}\right)}\left(F_{0}^{\prime}+m G_{0}\right)-\Theta_{1}, \\
& G_{1}^{\prime \prime}+G_{1}^{\prime}=\frac{M}{\left(1+m^{2}\right)}\left(G_{0}-m F_{0}^{\prime}\right), \\
& \Theta_{1}^{\prime \prime}+\operatorname{Pr} \Theta_{1}^{\prime}=0
\end{aligned}
$$


subject to the following boundary conditions

$$
\begin{aligned}
& F_{1}(0)=F_{1}^{\prime}(0)=0, \quad G_{1}(0)=0, \quad \Theta_{1}(0)=0, \\
& F_{1}^{\prime}(\infty)=G_{1}(\infty)=\Theta_{1}(\infty)=0 .
\end{aligned}
$$

The equations for $k \geq 2$ are given as;

$$
\begin{aligned}
& F_{k}^{\prime \prime \prime}+F_{k}^{\prime \prime}+\Theta_{k}=\frac{M}{\left(1+m^{2}\right)} F_{k-1}^{\prime}+\frac{M}{\left(1+m^{2}\right)} m G_{k-1} \\
& -\frac{1}{2}\left[\sum_{n=0}^{k-2} F_{k-2-n}^{\prime} n F_{n}^{\prime}-\sum_{n=0}^{k-2} F_{k-2-n}^{\prime \prime} n F_{n}\right] \\
& G_{k}^{\prime \prime}+G_{k}^{\prime}=\frac{M}{\left(1+m^{2}\right)} G_{k-1}-\frac{M}{\left(1+m^{2}\right)} m F_{k-1}^{\prime}, \\
& -\frac{1}{2}\left[\sum_{n=0}^{k-2} F_{k-2-n}^{\prime} n G_{n}-\sum_{n=0}^{k-2} G_{k-2-n}^{\prime} n F_{n}\right] \\
& \Theta_{k}^{\prime \prime}+\operatorname{Pr} \Theta_{k}^{\prime}=-\frac{1}{2} \operatorname{Pr}\left[\sum_{n=0}^{k-2} F_{k-2-n}^{\prime} n \Theta_{n}-\sum_{n=0}^{k-2} \Theta_{k-2-n}^{\prime} n F_{n}\right]
\end{aligned}
$$

subject to the following boundary conditions

$$
\begin{aligned}
& F_{k}(0)=F_{k}^{\prime}(0)=0, \quad G_{k}(0)=0, \quad \Theta_{k}(0)=0, \\
& F_{k}^{\prime}(\infty)=G_{k}(\infty)=\Theta_{k}(\infty)=0 .
\end{aligned}
$$

Solving equations (22 - 28) analytically yields:

$$
\begin{aligned}
& F_{0}(\bar{\eta})=\frac{1}{P r^{2}}+\frac{e^{-\bar{\eta}}}{\operatorname{Pr}(1-\operatorname{Pr})}-\frac{e^{-\operatorname{Pr} \bar{\eta}}}{\operatorname{Pr}^{2}(1-\operatorname{Pr})}, \\
& G_{0}(\bar{\eta})=0 \\
& \Theta_{0}(\bar{\eta})=e^{-\operatorname{Pr} \bar{\eta}},
\end{aligned}
$$

$F_{1}(\bar{\eta})=\frac{M\left[e^{P r \bar{\eta}}-e^{-\bar{\eta}}\left(\operatorname{Pr}+(-1+\operatorname{Pr})\left(e^{\bar{\eta}}\left(-1+\operatorname{Pr}^{2}\right)-\operatorname{Pr}^{2}(1+\bar{\eta})\right)\right)\right]}{\left(1+m^{2}\right)(-1+P r)^{2} \operatorname{Pr}^{3}}$

$G_{1}(\bar{\eta})=\frac{m M\left[e^{-\operatorname{Pr} \bar{\eta}}+e^{\bar{\eta}}\left(-1-\operatorname{Pr} \bar{\eta}+\operatorname{Pr}^{2} \bar{\eta}\right)\right]}{\left(1+m^{2}\right)(-1+\operatorname{Pr})^{2} \operatorname{Pr}^{2}}$,

$\Theta_{1}(\bar{\eta})=0$.

The Chebyshev spectral collocation method is then applied to integrate $(30-32)$. It is important to note that the spectral method is based on the Chebyshev polynomials defined on the domain $[-1,1]$ by

$$
T_{l}(x)=\cos \left[l \cos ^{-1}(x)\right] .
$$

Before using the spectral method, it is necessary to first transform the physical domain on which the governing equation is defined to the region $[-1,1]$ where the spectral method can then be applied. This can be done with the aid of the domain truncation procedure, the problem is solved in the interval $[0, L]$ in place of $[0, \infty)$, where $L$ is the scaling parameter taken to be large. The transformation $x=\frac{2 \bar{\eta}}{L}-1, \quad-1 \leq x \leq 1$, is used to map the domain $[0, L]$ to $[-1,1]$. The Gauss-lobatto collocation points Trefethen (2000) are used to define the Chebyshev nodes $[-1,1]$ as;

$$
x j=\cos \left(\frac{\pi j}{N_{x}}\right), \quad-1 \leq x \leq 1, \quad j=0,1,2 \ldots, N_{x},
$$

where $\left(N_{x}+1\right)$ is the total number of collocation points.

The basic idea behind the spectral collocation method is the introduction of the chebyshev differential matrix $\mathbf{D}$ (see for example, Trefethen (2000), Canuto et al. (1988)). The chebyshev differential matrix $\mathbf{D}$ is used to approximate the derivatives of the unknown variables $F_{k}(\bar{\eta}), G_{k}(\bar{\eta}), \Theta_{k}(\bar{\eta})$ at the collocation points as the matrix vector product

$$
\begin{gathered}
\left.\frac{d F_{k}}{d \bar{\eta}}\right|_{\bar{\eta}=\bar{\eta} j}=\sum_{l=0}^{N_{x}} \mathbf{D}_{j l} F_{k}\left(x_{l}\right)=\mathbf{D} \mathbf{F}_{k}, \quad j=0,1, \ldots, N_{x}, \\
\left.\frac{d G_{k}}{d \bar{\eta}}\right|_{\bar{\eta}=\bar{\eta} j}=\sum_{l=0}^{N_{x}} \mathbf{D}_{j l} G_{k}\left(x_{l}\right)=\mathbf{D G}_{k}, \quad j=0,1, \ldots, N_{x}, \\
\left.\frac{d \Theta_{k}}{d \bar{\eta}}\right|_{\bar{\eta}=\bar{\eta} j}=\sum_{l=0}^{N_{x}} \mathbf{D}_{j l} \Theta_{k}\left(x_{l}\right)=\mathbf{D} \Theta_{k}, \quad j=0,1, \ldots, N_{x},
\end{gathered}
$$

where $\left(N_{x}+1\right)$ is the number of collocation points, $\mathbf{D}=2 D / L$, and

$$
\begin{aligned}
& \mathbf{F}_{k}=\left[F_{k}\left(x_{0}\right), F_{k}\left(x_{1}\right), \ldots, F_{k}\left(x_{N_{x}}\right)\right]^{T}, \\
& \mathbf{G}_{k}=\left[G_{k}\left(x_{0}\right), G_{k}\left(x_{1}\right), \ldots, G_{k}\left(x_{N_{x}}\right)\right]^{T}, \\
& \boldsymbol{\Theta}_{k}=\left[\Theta_{k}\left(x_{0}\right), \Theta_{k}\left(x_{1}\right), \ldots, \Theta_{k}\left(x_{N_{x}}\right)\right]^{T},
\end{aligned}
$$

is the vector function at the collocation points. We obtain the higher order derivatives as powers of $\mathbf{D}$, that is;

$$
\mathbf{F}_{k}^{(p)}=\mathbf{D}^{p} \mathbf{F}_{k}, \quad \mathbf{G}_{k}^{(p)}=\mathbf{D}^{p} \mathbf{G}_{k}, \quad \Theta_{k}^{(p)}=\mathbf{D}^{p} \boldsymbol{\Theta}_{k},
$$

where $p$ is the order of the derivatives. The matrix $D$ is of size $\left(N_{x}+1\right) \times\left(N_{x}+1\right)$ and its entries are defined in Trefethen (2000); Canuto et al. (1988) as;

$$
\begin{aligned}
D_{j l} & =\frac{c_{j}}{c_{l}} \frac{(-1)^{j+l}}{\tau_{j}-\tau_{l}} \quad j \neq l ; j, l=0,1,2, N, \\
D_{l l} & =-\frac{\tau_{l}}{2\left(1-\tau_{l}^{2}\right)} \quad 1 \leq j=l \leq N-1, \\
D_{00} & =\frac{2 N^{2}+1}{6}=-D_{N_{x} N_{x}},
\end{aligned}
$$

with

$$
c_{l}=\left\{\begin{array}{c}
2, \quad l=0, N_{x} \\
-1, \quad-1 \leq l \leq N_{x}-1 .
\end{array}\right.
$$

Substituting (42 - 48) in (30 - 32) yields

$$
\mathbf{A}_{1, k-1} \mathbf{F}_{k}=\mathbf{B}_{1, k-1}, \quad \mathbf{A}_{2, k-1} \mathbf{G}_{k}=\mathbf{B}_{2, k-1}, \quad \mathbf{A}_{3, k-1} \boldsymbol{\Theta}_{k}=\mathbf{B}_{3, k-1},
$$

subject to the following boundary conditions

$$
\begin{aligned}
& \sum_{l=0}^{N_{x}} \mathbf{D}_{0 l} F_{k}\left(x_{l}\right)=0, \quad \sum_{l=0}^{N_{x}} \mathbf{D}_{N_{x} l} F_{k}\left(x_{l}\right)=0, \quad F_{k}\left(x_{N_{x}}\right)=0, \\
& G_{k}\left(x_{N_{x}}\right)=0, \quad G_{k}\left(x_{0}\right)=0 \\
& \Theta_{k}\left(x_{N_{x}}\right)=0, \quad \Theta_{k}\left(x_{0}\right)=0
\end{aligned}
$$

where $\mathbf{A}_{1, k-1}, \mathbf{A}_{2, k-1}, \mathbf{A}_{3, k-1}, \mathbf{B}_{1, k-1}, \mathbf{B}_{2, k-1}$, and $\mathbf{B}_{3, k-1}$ are defined as;

$$
\begin{aligned}
& \mathbf{A}_{1, k-1}=\mathbf{D}^{3}+\mathbf{D}^{2}, \quad \mathbf{A}_{2, k-1}=\mathbf{D}^{2}+\mathbf{D}, \quad \mathbf{A}_{3, k-1}=\mathbf{D}^{2}+\mathbf{D}, \\
& \mathbf{B}_{1, k-1}=\frac{M}{\left(1+m^{2}\right)}\left(\mathbf{D} \mathbf{F}_{k-1}\right)+\frac{M}{\left(1+m^{2}\right)} m \mathbf{G}_{k-1}-\mathbf{\Theta}_{k}+\text { SumF }, \\
& \mathbf{B}_{2, k-1}=\frac{M}{\left(1+m^{2}\right)} \mathbf{G}_{k-1}-\frac{M}{\left(1+m^{2}\right)} m\left(\mathbf{D F}_{k-1}\right)+\text { SumG, } \\
& \mathbf{B}_{3, k-1}=\operatorname{Sum} \boldsymbol{\Theta},
\end{aligned}
$$

where SumF, SumG and $\operatorname{Sum} \Theta$ are defined as;

$$
\begin{aligned}
& \text { SumF }=-\frac{1}{2} \sum_{n=0}^{k-2}\left[\left(\mathbf{D F} \mathbf{F}_{k-2-n}\right)\left(n \mathbf{D F} \mathbf{F}_{n}\right)-\left(\mathbf{D}^{2} \mathbf{F}_{k-2-n}\right)\left(n \mathbf{F}_{n}\right)\right], \\
& \text { SumG }=-\frac{1}{2} \sum_{i=0}^{k-2}\left[\left(\mathbf{D F}{ }_{k-2-n}\right)\left(n \mathbf{G}_{n}\right)-\left(\mathbf{D} \mathbf{G}_{k-2-n}\right)\left(n \mathbf{F}_{n}\right)\right], \\
& \operatorname{Sum} \Theta=-\frac{1}{2} \operatorname{Pr} \sum_{i=0}^{k-2}\left[\left(\mathbf{D F}_{k-2-n}\right)\left(n \boldsymbol{\Theta}_{n}\right)-\left(\mathbf{D} \Theta_{k-2-n}\right)\left(n \mathbf{F}_{n}\right)\right],
\end{aligned}
$$


The boundary condition (52) is imposed on the first, $N_{x}$ th row (second from the last row) and $\left(N_{x}+1\right)$ st row (last row) rows and first and last columns of $\mathbf{A}_{1, k-1} \mathbf{F}_{k}=\mathbf{B}_{1, k-1}$ to obtain subject to the following boundary conditions

$$
F_{0}(0)=F_{0}^{\prime}(0)=0, \quad \Theta_{0}(0)=1, \quad F_{0}^{\prime}(\infty)=\Theta_{0}(\infty)=0 .
$$

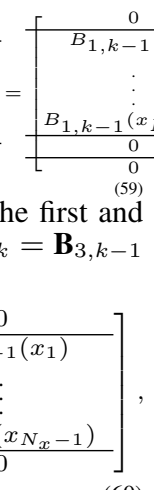

(60)

and

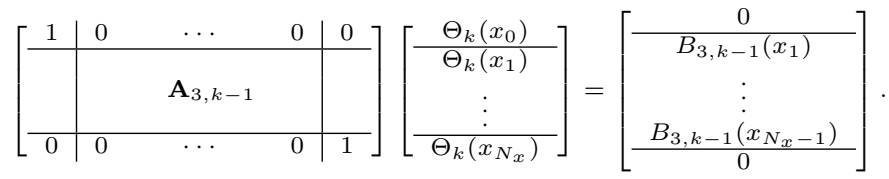

(61)

Therefore, starting from a known $F_{0}, \Theta_{0}, \Phi_{0}$, the solutions $F_{k}, G_{k}, \Theta_{k}$, for $k \geq 2$ can be obtained from equations (59 - 61) as;

$$
\mathbf{F}_{k}=\mathbf{A}_{1, k-1}^{-1} \mathbf{B}_{1, k-1}, \quad \mathbf{G}_{k}=\mathbf{A}_{2, k-1}^{-1} \mathbf{B}_{2, k-1}, \quad \boldsymbol{\Theta}_{k}=\mathbf{A}_{3, k-1}^{-1} \mathbf{B}_{3, k-1} .
$$

\subsection{Large parameter Spectral Perturbation Method Solution for large $(\xi)$ for Equations (6 - 7)}

To solve equations ( $6-7)$, attention shall be given to the solutions of equations (6 and 7) when $\xi$ is large. An order of magnitude of different terms in equations (6 and 7) shows that the largest terms in (6) are $f^{\prime \prime \prime}$ and $\xi f^{\prime \prime}$, and $\theta^{\prime \prime}$ and $\xi \theta^{\prime}$ in (7). Both terms have to be balanced in the respective equations and the only way to do this is to assume that $\eta$ to be small and its derivatives are large. Given that $\theta=O(1)$ as $\xi \rightarrow \infty$, it is necessary to find appropriate scaling for $f$ and $\eta$. On balancing the $f^{\prime \prime \prime}$ ,$\theta$ and $\xi f^{\prime \prime}$ terms in (6), it is found that $\eta=O\left(\xi^{-1}\right)$ and $f=O\left(\xi^{-3}\right)$ as $\xi \rightarrow \infty$. Therefore, the following transformations are introduced to switch the equations for small $\xi$ to those for large $\xi$;

$$
f=\xi^{-3} F(\xi, \bar{\eta}), \quad \bar{\eta}=\xi \eta, \quad \theta=\Theta(\xi, \bar{\eta}) .
$$

Substituting these transformations given in (63) into equations (6 - 7), we obtain the following equations:

$F^{\prime \prime \prime}+F^{\prime \prime}+\Theta+(1+n) \xi^{-4} F F^{\prime \prime}-n \xi^{-4} F^{\prime 2}=\frac{1-n}{4} \xi^{-3}\left[F^{\prime} \frac{\partial F^{\prime}}{\partial \xi}-F^{\prime \prime} \frac{\partial F}{\partial \xi}\right]$

$\Theta^{\prime \prime}+\operatorname{Pr} \Theta^{\prime}+(1+n) \operatorname{Pr} \xi^{-4} F \Theta^{\prime}-n \operatorname{Pr}^{-4} F^{\prime} \Theta=\frac{1-n}{4} \operatorname{Pr} \xi^{-3}\left[F^{\prime} \frac{\partial \Theta}{\partial \xi}-\right.$

The corresponding boundary conditions are given by ;

$F(\xi, 0)=F^{\prime}(\xi, 0)=0, \quad \Theta(\xi, 0)=1, \quad F^{\prime}(\xi, \infty)=\Theta(\xi, \infty)=0$.

Since $\xi$ is large, solutions of equations $(64-65)$ is obtained using the spectral perturbation method. Hence, we expand the functions $F(\xi, \bar{\eta})$, and $\Theta(\xi, \bar{\eta})$ in powers of $\xi^{-4}$ as given below;

$$
F(\xi, \bar{\eta})=\sum_{k=0}^{\infty} \xi^{-4 k} F_{k}(\bar{\eta}), \quad \Theta(\xi, \bar{\eta})=\sum_{k=0}^{\infty} \xi^{-4 k} \Theta_{k}(\bar{\eta}) .
$$

Substituting equation (67) into equations (64) and (65) and then equating the coefficients of like powers of $\xi$, we obtain the equations for $k=0$ as;

$$
F_{0}^{\prime \prime \prime}+F_{0}^{\prime \prime}+\Theta_{0}=0, \quad \Theta_{0}^{\prime \prime}+\operatorname{Pr} \Theta_{0}^{\prime}=0,
$$

The equations for $k \geq 1$ are given as;

$$
\begin{aligned}
& F_{k}^{\prime \prime \prime}+F_{k}^{\prime \prime}+\Theta_{k}=n \sum_{i=0}^{k-1} F_{k-1-i}^{\prime} F_{i}^{\prime}-(1+n) \sum_{i=0}^{k-1} F_{k-1-i} F_{i}^{\prime \prime}, \\
& +(1-n)\left[\sum_{i=0}^{k-1} F_{k-1-i}^{\prime \prime} i F_{i}-\sum_{i=0}^{k-1} F_{k-1-i}^{\prime} i F_{i}^{\prime}\right] \\
& \Theta_{k}^{\prime \prime}+\operatorname{Pr} \Theta_{k}^{\prime}=n \operatorname{Pr} \sum_{i=0}^{k-1} F_{k-1-i}^{\prime} \Theta_{i}-(1+n) \operatorname{Pr} \sum_{i=0}^{k-1} F_{k-1-i} \Theta_{i}^{\prime}, \\
& +(1-n) \operatorname{Pr}\left[\sum_{i=0}^{k-1} \Theta_{k-1-i}^{\prime} i F_{i}-\sum_{i=0}^{k-1} F_{k-1-i}^{\prime} i \Theta_{i}\right]
\end{aligned}
$$

subject to the following boundary conditions

$$
F_{k}(0)=F_{k}^{\prime}(0)=0, \quad \Theta_{k}(0)=0, \quad F_{k}^{\prime}(\infty)=\Theta_{k}(\infty)=0 .
$$

Since the left hand side of of the higher order perturbation equations (71) - (72) are linear, we therefore apply the Chebyshev spectral collocation method described in the previous section to integrate equations (71 - 72). We remark that the equations at $k=0$ and $k=1$, can be solved analytically. The equations corresponding to $k=1$ is;

$$
\begin{aligned}
& F_{1}^{\prime \prime \prime}+F_{1}^{\prime \prime}+(1+n) F_{0} F_{0}^{\prime \prime}-n F_{0}^{2}=-\Theta_{1}, \\
& \Theta_{1}^{\prime \prime}+\operatorname{Pr} \Theta_{1}^{\prime}+(1+n) \operatorname{Pr} F_{0} \Theta_{0}^{\prime}-n \operatorname{Pr} F_{0}^{\prime} \Theta_{0}=0,
\end{aligned}
$$

subject to the following boundary conditions:

$$
F_{1}(0)=F_{1}^{\prime}(0)=0, \quad \Theta_{1}(0)=0, \quad F_{1}^{\prime}(\infty)=\Theta_{1}(\infty)=0 .
$$

On solving equations (68) and (73) analytically, their solutions were obtained as:

$$
\begin{aligned}
& F_{0}(\bar{\eta})=\frac{1}{\operatorname{Pr}^{2}}+\frac{1}{\operatorname{Pr}(1-\operatorname{Pr})} e^{-\bar{\eta}}-\frac{1}{\operatorname{Pr}^{2}(1-\operatorname{Pr})} e^{-\operatorname{Pr} \bar{\eta}} \\
& \Theta_{0}(\bar{\eta})=e^{-\operatorname{Pr} \bar{\eta}}
\end{aligned}
$$

$$
F_{1}(\bar{\eta})=\frac{(1+P r)-2 P r^{3}+2 P r^{2}(1-P r) n}{2 P r^{4}\left(1-P r^{2}\right)}-\frac{3(1+n)}{\operatorname{Pr}^{4}(1-P r)}+\frac{1+P r+P r^{2}}{8 \operatorname{Pr}^{4}(1-P r)^{2}},
$$$$
+\frac{P r-(1-P r) n}{\left(1-\operatorname{Pr}^{2}\right)(1-P r)^{2}}+\frac{(1+n)\left(1+\operatorname{Pr}^{2}\right)-2 \operatorname{Prn}}{\operatorname{Pr}^{3}\left(1-\operatorname{Pr}^{2}\right)^{2}},
$$$$
+\left[\frac{(1+P r)-2 P r^{3}+2 P r^{2}(1-P r) n}{2 P r^{3}(1-P r)^{2}(1+P r)}-\frac{3(1+n)}{P r^{3}(1-P r)}\right] e^{-\bar{\eta}},
$$$$
+\left[\frac{1+P r}{8 P^{3}(1-P r)^{2}(1-2 P r)}-\frac{P r-(1-P r) n}{P r\left(1-P r^{2}\right)(1+P r)}\right] e^{-\bar{\eta}},
$$

$\left.\Theta^{\prime} \frac{\partial F}{\partial \xi}\right]+\left[\frac{(1+n)\left(1+P r^{2}\right)-2 P r n}{P r^{4}\left(1-P r^{2}\right)^{2}(1+P r)}-\frac{1}{2 P r^{2}(1-P r)^{2}}\right] e^{-\bar{\eta}}$

$+\left[-\frac{(1+P r)-2 P r^{3}+2 P r^{2}(1-P r) n}{2 \operatorname{Pr}^{4}(1-P r)^{2}(1+P r)}-\frac{3(1+n)}{\operatorname{Pr}^{4}(1-P r)}\right] e^{-P r \bar{\eta}}$,

$+\left[\frac{1+n}{\operatorname{Pr}^{3}(1-P r)} \bar{\eta}\right] e^{-P r \bar{\eta}}-\left[\frac{1+n}{\operatorname{Pr}^{3}(1-P r)}\right] \bar{\eta} e^{-\bar{\eta}}$,

$+\left[\frac{P r-(1-P r) n}{\operatorname{Pr}\left(1-\operatorname{Pr}^{2}\right)(1+P r)^{2}}-\frac{(1+n)\left(1+P r^{2}\right)-2 P r n}{\operatorname{Pr}^{4}\left(1-\operatorname{Pr}^{2}\right)^{2}}\right] e^{-(1+P r) \bar{\eta}}$,

$-\left[\frac{1+P r}{8 \operatorname{Pr}^{4}(1-P r)^{2}(1-2 P r)}\right] e^{-2 P r \bar{\eta}}+\frac{e^{-2 \bar{\eta}}}{4 \operatorname{Pr}^{2}(1-P r)^{2}}$.

$\Theta_{1}(\bar{\eta})=\left[\frac{(1+P r)-2 P r^{3}+2 P r^{2}(1-P r) n}{2 P r^{2}\left(1-P r^{2}\right)}-\frac{\bar{\eta}+n \bar{\eta}}{P r}\right] e^{-P r \bar{\eta}}$

$+\left[\frac{P r-(1-P r) n}{1-P r^{2}}\right] e^{-(1-P r) \bar{\eta}}-\frac{e^{-2 P r \bar{\eta}}}{P r^{2}(1-P r)}$. 
The differential matrix $D$ is used to approximate the derivatives of the unknown variables $F_{k}(\bar{\eta}), \Theta_{k}(\bar{\eta}$ at the collocation points and are defined as;

$$
\begin{aligned}
& \left.\frac{d F_{k}}{d \bar{\eta}}\right|_{\bar{\eta}=\bar{\eta} j}=\sum_{l=0}^{N_{x}} \mathbf{D}_{j l} F_{k}\left(x_{l}\right)=\mathbf{D F} \mathbf{F}_{k}, \quad j=0,1, \ldots, N_{x}, \\
& \left.\frac{d \Theta_{k}}{d \bar{\eta}}\right|_{\bar{\eta}=\bar{\eta} j}=\sum_{l=0}^{N_{x}} \mathbf{D}_{j l} \Theta_{k}\left(x_{l}\right)=\mathbf{D} \Theta_{k}, \quad j=0,1, \ldots, N_{x},
\end{aligned}
$$

where $N_{x}+1$ is the total number of collocation points, $\mathbf{D}=2 D / L$, and

$$
\begin{aligned}
& \mathbf{F}_{k}=\left[F_{k}\left(x_{0}\right), F_{k}\left(x_{1}\right), \ldots, F_{k}\left(x_{N x}\right)\right]^{T}, \\
& \Theta_{k}=\left[\Theta_{k}\left(x_{0}\right), \Theta_{k}\left(x_{1}\right), \cdots, \Theta_{k}\left(x_{N x}\right)\right]^{T},
\end{aligned}
$$

are the vector functions at the collocation points. The matrix $D$ is of size $\left(N_{x}+1\right) \times\left(N_{x}+1\right)$ and its entries are defined in the previous section. Substituting $(78-80)$ in $(70-71)$ gives

$$
\mathbf{A}_{1, k-1} \mathbf{F}_{k}=\mathbf{B}_{1, k-1}, \quad \mathbf{A}_{2, k-1} \boldsymbol{\Theta}_{k}=\mathbf{B}_{2, k-1},
$$

subject to the boundary conditions

$$
\begin{aligned}
& \sum_{l=0}^{N_{x}} \mathbf{D}_{0 k} F_{k}\left(\bar{\eta}_{k}\right)=0, \quad \sum_{l=0}^{N_{x}} \mathbf{D}_{N x k} F_{k}\left(\bar{\eta}_{k}\right)=0, \quad F_{k}\left(\bar{\eta}_{N x}\right)=0, \\
& \Theta_{k}\left(\eta_{N x}\right)=0, \quad \Theta_{k}\left(\eta_{0}\right)=0 .
\end{aligned}
$$

The coefficients $\mathbf{A}_{1, k-1}, \mathbf{A}_{2, k-1}, \mathbf{B}_{1, k-1}, \mathbf{B}_{2, k-1}$, are defined as;

$$
\begin{gathered}
\mathbf{A}_{1, k-1}=\mathbf{D}^{3}+\mathbf{D}^{2}+\boldsymbol{\Theta}_{k}, \quad \mathbf{A}_{2, k-1}=\mathbf{D}^{2}+\operatorname{Pr} \mathbf{D}, \\
\mathbf{B}_{1, k-1}=n \sum_{i=0}^{k-1}\left(\mathbf{D} \mathbf{F}_{k-1-i}\right)\left(\mathbf{D} \mathbf{F}_{i}\right)-(1+n) \sum_{i=0}^{k-1} \mathbf{F}_{k-1-i}\left(\mathbf{D}^{2} \mathbf{F}_{i}\right) \\
+(1-n)\left[\sum_{i=0}^{k-1}\left(\mathbf{D}^{2} \mathbf{F}_{k-1-i}\right)\left(i \mathbf{F}_{i}\right)-\sum_{i=0}^{k-1}\left(\mathbf{D} \mathbf{F}_{k-1-i}\right)\left(i \mathbf{D} \mathbf{F}_{i}\right)\right], \\
\mathbf{B}_{2, k-1}=n \operatorname{Pr} \sum_{i=0}^{k-1}\left(\mathbf{D} \mathbf{F}_{k-1-i}\right)\left(\mathbf{F}_{i}\right)-(1+n) \operatorname{Pr} \sum_{i=0}^{k-1} \mathbf{F}_{k-1-i}\left(\mathbf{D} \boldsymbol{\Theta}_{i}\right) \\
+(1-n) \operatorname{Pr}\left[\sum_{i=0}^{k-1}\left(\mathbf{D \Theta}_{k-1-i}\right)\left(i \mathbf{F}_{i}\right)-\sum_{i=0}^{k-1}\left(\mathbf{D} \mathbf{F}_{k-1-i}\right)\left(i \mathbf{\Theta}_{i}\right)\right]
\end{gathered}
$$

The boundary condition (82) is imposed on the first, $N_{x}$ th row (second from the last row) and $\left(N_{x}+1\right)$ st row (last row) rows and first and last columns of $\mathbf{A}_{1, k-1} \mathbf{F}_{k}=\mathbf{B}_{1, k-1}$, while the boundary conditions (83) is imposed on the first and last rows and columns of $\mathbf{A}_{2, k-1} \boldsymbol{\Theta}_{k}=\mathbf{B}_{2, k-1}$, . Thus, starting from a known $F_{0}$, and $\Theta_{0}$, the solutions $F_{k}$, and $\Theta_{k}$, for $k \geq 1$ can be obtained as;

$$
F_{k}=\mathbf{A}_{1, k-1}^{-1} \mathbf{B}_{1, k-1}, \quad \Theta_{k}=\mathbf{A}_{2, k-1}^{-1} \mathbf{B}_{2, k-1} .
$$

\subsection{Large Parameter Spectral Perturbation Method Solution for large $(\xi)$ for Equations (10 - 11)}

To find the solutions of equations $(10$ - 11) along with boundary conditions 12 , attention shall be given to the solutions of equations $(10-11)$ when $\xi$ is large. An order of magnitude of various terms carried out in equations $(10-11)$ depicts that the largest in (10) are $f^{\prime \prime \prime}$ and $\xi f^{\prime \prime}$, and $\phi^{\prime \prime}$ and $\xi \phi^{\prime}$ in (11). Both terms have to be balanced in the respective equations and the only way to do this is to assume that $\eta$ is small and its derivatives are large. Given that $\phi=O\left(\xi^{-1}\right)$ as $\xi \rightarrow \infty$, it is essential to find appropriate scaling for $f$ and $\eta$. On balancing the $f^{\prime \prime \prime}, \phi$ and $\xi f^{\prime \prime}$ terms in (10), it is found that $\eta=O\left(\xi^{-1}\right)$ and $f=O\left(\xi^{-4}\right)$ as $\xi \rightarrow \infty$. Therefore, the following transformations are introduced to switch the equations for small $\xi$ to those for large $\xi$;

$$
f=\xi^{-4} F(\xi, \bar{\eta}), \quad \bar{\eta}=\xi \eta, \quad \phi=\xi^{-1} \Phi(\eta, \bar{\eta}) .
$$

Substituting these transformations given in (88) into equations (10 - 11), we obtain the following equations:

$$
\begin{aligned}
& F^{\prime \prime \prime}+F^{\prime \prime}+\Phi+(1+m) \xi^{-5} F F^{\prime \prime}-m \xi^{-5} F^{\prime 2}, \\
& =\frac{1-m}{5} \xi^{-4}\left[F^{\prime} \frac{\partial F^{\prime}}{\partial \xi}-F^{\prime \prime} \frac{\partial F}{\partial \xi}\right], \\
& \Phi^{\prime \prime}+\operatorname{Pr} \Phi^{\prime}+(1+m) \operatorname{Pr} \xi^{-5} F \Phi^{\prime}-m \operatorname{Pr} \xi^{-5} F^{\prime} \Phi, \\
& =\frac{1-m}{5} \operatorname{Pr}^{-4}\left[F^{\prime} \frac{\partial \Phi}{\partial \xi}-\Phi^{\prime} \frac{\partial F}{\partial \xi}\right] .
\end{aligned}
$$

The corresponding boundary conditions are given as;

$F(\xi, 0)=F^{\prime}(\xi, 0)=0, \quad \Phi^{\prime}(\xi, 0)=-1, \quad F^{\prime}(\xi, \infty)=\Phi(\xi, \infty)=0$.

The functions $F(\xi, \bar{\eta})$, and $\Phi(\xi, \bar{\eta})$ are expanded in powers of $\xi^{-5}$ and written below as;

$$
\begin{aligned}
& F(\xi, \bar{\eta})=\sum_{k=0}^{\infty} \xi^{-5 k} F_{k}(\bar{\eta}), \\
& \Phi(\xi, \bar{\eta})=\sum_{k=0}^{\infty} \xi^{-5 k} \Phi_{k}(\bar{\eta}) .
\end{aligned}
$$

Substituting equations (92) - (93) into equations (89) - (90) and then balancing terms of same orders of $\xi$, we obtain the equations for $k=0$ as;

$$
F_{0}^{\prime \prime \prime}+F_{0}^{\prime \prime}=-\Phi_{0}, \quad \Phi_{0}^{\prime \prime}+\operatorname{Pr} \Phi_{0}^{\prime}=0,
$$

subject to the following boundary conditions

$$
F_{0}(0)=F_{0}^{\prime}(0)=0, \quad \Phi_{0}^{\prime}(0)=-1, \quad F_{0}^{\prime}(\infty)=\Phi_{0}(\infty)=0 .
$$

The equations for $k \geq 1$ are given as;

$$
\begin{aligned}
& F_{k}^{\prime \prime \prime}+F_{k}^{\prime \prime}+\Phi_{k}=m \sum_{i=0}^{k-1} F_{k-1-i}^{\prime} F_{i}^{\prime}-(1+m) \sum_{i=0}^{k-1} F_{k-1-i} F_{i}^{\prime \prime} \\
& +(1-m)\left[\sum_{i=0}^{k-1} F_{k-1-i}^{\prime \prime} i F_{i}-\sum_{i=0}^{k-1} F_{k-1-i}^{\prime} i F_{i}^{\prime}\right] \\
& \Phi_{k}^{\prime \prime}+\operatorname{Pr} \Phi_{k}^{\prime}=m \operatorname{Pr} \sum_{i=0}^{k-1} F_{k-1-i}^{\prime} \Phi_{i}-(1+m) \operatorname{Pr} \sum_{i=0}^{k-1} F_{k-1-i} \Phi_{i}^{\prime} \\
& +(1-m) \operatorname{Pr}\left[\sum_{i=0}^{k-1} \Phi_{k-1-i}^{\prime} i F_{i}-\sum_{i=0}^{k-1} F_{k-1-i}^{\prime} i \Phi_{i}\right]
\end{aligned}
$$

subject to the following boundary conditions

$$
F_{k}(0)=F_{k}^{\prime}(0)=0, \quad \Phi_{k}^{\prime}(0)=0, \quad F_{k}^{\prime}(\infty)=\Phi_{k}(\infty)=0 .
$$

The equations corresponding to $k=1$ is;

$$
\begin{aligned}
& F_{1}^{\prime \prime \prime}+F_{1}^{\prime \prime}+(1+m) F_{0} F_{0}^{\prime \prime}-m F_{0}^{\prime 2}=-\Phi_{1}, \\
& \Phi_{1}^{\prime \prime}+\operatorname{Pr} \Phi_{1}^{\prime}+(1+m) \operatorname{Pr} F_{0} \Phi_{0}^{\prime}-n \operatorname{Pr} F_{0}^{\prime} \Phi_{0}=0,
\end{aligned}
$$

subject to the boundary conditions;

$$
F_{1}(0)=F_{1}^{\prime}(0)=0, \quad \Phi_{1}^{\prime}(0)=0, \quad F_{1}^{\prime}(\infty)=\Phi_{1}(\infty)=0 .
$$


We remark the analytical solutions obtained on solving equations (94) and (97) are:

$$
\begin{aligned}
& F_{0}(\bar{\eta})=\frac{1}{P r^{3}}+\frac{e^{-\bar{\eta}}}{P r^{2}(1-P r)}-\frac{e^{-P r \bar{\eta}}}{P r^{3}(1-P r)}, \\
& \Phi_{0}(\bar{\eta})=\frac{e^{-P r \bar{\eta}}}{\operatorname{Pr}} \\
& F_{1}(\bar{\eta})=\frac{1-(1+m)(4-P r)}{P r^{6}}+\frac{P r-m(1-P r)}{\operatorname{Pr}^{2}(1-P r)^{2}\left(1-P r^{2}\right)}, \\
& -\frac{(1+m)\left(1+P r^{2}\right)-2 m P r}{P r^{5}\left(1-P r^{2}\right)^{2}}+\frac{1+P r+2 P r^{2}}{8 \operatorname{Pr}^{6}(1-P r)^{2}}, \\
& +\left[\frac{1-(1+m)(4-P r)}{\operatorname{Pr}^{5}(1-\operatorname{Pr})}\right] e^{-\bar{\eta}}-\left[\frac{\operatorname{Pr}-m(1-P r)}{\operatorname{Pr}^{3}(1+\operatorname{Pr})\left(1-\operatorname{Pr}^{2}\right)}\right] e^{-\bar{\eta}}, \\
& +\left[\frac{1}{2 P r^{4}(1-P r)^{2}}-\frac{(1+m)\left(1+P r^{2}\right)-2 m P r}{P r^{6}(1-P r)^{2}(1+P r)}\right] e^{-\bar{\eta}}, \\
& +\left[\frac{1+P r}{4 \operatorname{Pr}^{5}(1-P r)^{2}(1-2 P r)}\right] e^{-\bar{\eta}}+\left[\frac{-\bar{\eta}+(1+m)(-2 \bar{\eta}-\operatorname{Pr} \bar{\eta}-1)}{\operatorname{Pr}^{6}(1-\operatorname{Pr}) \bar{\eta}}\right] e^{-\operatorname{Pr} \bar{\eta}} \\
& +\left[\frac{P r-m(1-P r)}{P r^{3}(1+P r)^{2}\left(1-P r^{2}\right)}\right] e^{-(1+P r) \bar{\eta}}, \\
& -\left[\frac{(1+m)\left(1+P r^{2}\right)-2 m P r}{P r^{6}\left(1-P r^{2}\right)^{2}}\right] e^{-(1+P r) \bar{\eta}}-\left[\frac{1+m}{P r^{5}(1-P r)} \bar{\eta}\right] e^{-\bar{\eta}}, \\
& -\left[\frac{1+\operatorname{Pr}}{8 \operatorname{Pr}^{6}(1+P r)^{2}(1-2 P r)}\right] e^{-2 P r \bar{\eta}}+\frac{e^{-2 \bar{\eta}}}{4 P r^{4}(1-P r)^{2}} \text {. } \\
& \Phi_{1}(\bar{\eta})=\left[\frac{1-(1+m)(1-\operatorname{Pr}+\operatorname{Pr} \bar{\eta})}{P r^{4}}\right] e^{-\operatorname{Pr} \bar{\eta}}, \\
& +\left[\frac{P r-m(1-P r)}{\operatorname{Pr}^{2}\left(1-\operatorname{Pr}^{2}\right)}\right] e^{-(1+P r) \bar{\eta}}-\frac{e^{-2 P r \bar{\eta}}}{2 \operatorname{Pr}^{4}(1-P r)} .
\end{aligned}
$$

Applying the Chebyshev spectral method on equations (95) and (96) gives:

$$
\mathbf{A}_{1, k-1} \mathbf{F}_{k}=\mathbf{B}_{1, k-1}, \quad \mathbf{A}_{2, k-1} \boldsymbol{\Phi}_{k}=\mathbf{B}_{2, k-1},
$$

subject to the boundary conditions

$$
\begin{aligned}
& \sum_{l=0}^{N_{x}} \mathbf{D}_{0 k} F_{k}\left(\bar{\eta}_{k}\right)=0, \quad \sum_{l=0}^{N_{x}} \mathbf{D}_{N x k} F_{k}\left(\bar{\eta}_{k}\right)=0, \quad F_{k}\left(\bar{\eta}_{N x}\right)=0 \\
& \Phi_{k}\left(\eta_{N x}\right)=0, \quad \Phi_{k}\left(\eta_{0}\right)=0 .
\end{aligned}
$$

The coefficients $\mathbf{A}_{1, k-1}, \mathbf{A}_{2, k-1}, \mathbf{B}_{1, k-1}, \mathbf{B}_{2, k-1}$, are defined as;

$$
\begin{gathered}
\mathbf{A}_{1, k-1}=\mathbf{D}^{3}+\mathbf{D}^{2} \quad \mathbf{A}_{2, k-1}=\mathbf{D}^{2}+\operatorname{Pr} \mathbf{D}, \\
\mathbf{B}_{1, k-1}=m \sum_{i=0}^{k-1}\left(\mathbf{D F}_{k-1-i}\right)\left(\mathbf{D} \mathbf{F}_{i}\right)-(1+m) \sum_{i=0}^{k-1} \mathbf{F}_{k-1-i}\left(\mathbf{D}^{2} \mathbf{F}_{i}\right) \\
+(1-m)\left[\sum_{i=0}^{k-1}\left(\mathbf{D}^{2} \mathbf{F}_{k-1-i}\right)\left(i \mathbf{F}_{i}\right)-\sum_{i=0}^{k-1}\left(\mathbf{D F}_{k-1-i}\right)\left(i \mathbf{D} \mathbf{F}_{i}\right)\right]-\boldsymbol{\Phi}_{k},
\end{gathered}
$$

$$
\begin{aligned}
& \mathbf{B}_{2, k-1}=m \operatorname{Pr} \sum_{i=0}^{k-1}\left(\mathbf{D F} \mathbf{F}_{k-1-n}\right)\left(\mathbf{\Phi}_{n}\right)-(1+m) \operatorname{Pr} \sum_{i=0}^{k-1} \mathbf{F}_{k-1-i}\left(\mathbf{D} \boldsymbol{\Phi}_{i}\right) \\
& +(1-m) \operatorname{Pr}\left[\sum_{i=0}^{k-1}\left(\mathbf{D} \boldsymbol{\Phi}_{k-1-i}\right)\left(i \mathbf{F}_{i}\right)-\sum_{i=0}^{k-1}\left(\mathbf{D F}_{k-1-i}\right)\left(i \boldsymbol{\Theta}_{i}\right)\right] .
\end{aligned}
$$

Thus, starting from a known $F_{0}$, and $\Phi_{0}$, the solutions $F_{k}$, and $\Phi_{k}$, for $k \geq 1$ can be obtained.

\section{RESULTS AND DISCUSSION}

In this section, the nonlinear systems of partial differential equations ( 1 $3),(6-7)$, and (10 - 11) were solve numerically using the large parameter spectral perturbation method (LSPM). Results were presented for the skin friction coefficient and Nusselt number for different physical parameters that are of interest to the flow model. The accuracy of the computed (LSPM) approximate numerical results were confirmed by comparing with other reported results from literature. The comparison was done in particular against the reported results of Saha et al. (2007) who solved equations (1-2) using regular perturbation method, asymptotic method solution, implicit finite difference method together with Keller-box scheme and local nonsimilarity method. In addition, comparison were also made against published results of Hossain and Paul (2001a,b) who used the finite difference method, series solution method and an asymptotic solution method to respectively, solve equations (6-7) and (10-11). Validation of the numerical solutions was further established by comparing the (LSPM) results with numerical approximate solutions obtained using the bivariate quasilinearisation method (BSQLM) as described by Motsa et al. (2014) and Motsa and Ansari (2015). We remark that the values of all physical parameters used in this study were chosen based on the values used in the published work of Hossain and Paul (2001a,b); Saha et al. (2007). The number of collocation points $N_{x}$ used was 60 if not stated. In order to further check the accuracy of the LSPM, we obtain the error norm of the approximate solution and the residual error. The error norm of the approximate solution can be defined as the difference between the approximate values of $F, \Theta$, and $\Phi$ at the next approximation level, which is given as;

$$
\begin{aligned}
& E_{F}=\left\|\mathbf{F}_{m+1}-\mathbf{F}_{m}\right\|_{\infty}, \\
& E_{\Theta}=\left\|\boldsymbol{\Theta}_{m+1}-\boldsymbol{\Theta}_{m}\right\|_{\infty}, \\
& E_{\Phi}=\left\|\boldsymbol{\Phi}_{m+1}-\boldsymbol{\Phi}_{m}\right\|_{\infty},
\end{aligned}
$$

where $F_{m+1}, \Theta_{m+1}$ and $\Phi_{m+1}$ are the previous approximation level and $F_{m}, \Theta_{m}$, and $\Phi_{m}$ are the current approximation level. Furthermore, to define the residual error, we assume that $x^{*}$ is an approximation to the solution $A x=b$, the residual $r=b-A x^{*}$. The residual error after $m$ approximation over all $r=0,1,2, \cdots, N_{x}$ can be defined as;

$$
\begin{gathered}
\operatorname{Res}(F)=\max _{0 \leq j \leq N_{x}}\left|\bar{N}_{F}\left[\mathbf{F}_{m}(\xi, \eta), \boldsymbol{\Theta}_{m}(\xi, \eta)\right]\right|, \\
\operatorname{Res}(\Theta)=\max _{0 \leq j \leq N_{x}}\left|\bar{N}_{\Theta}\left[\mathbf{F}_{m}(\xi, \eta),(\xi, \eta), \boldsymbol{\Theta}_{m}(\xi, \eta)\right]\right|, \\
\operatorname{Res}(\Phi)=\max _{0 \leq j \leq N_{x}}\left|\bar{N}_{\Theta}\left[\mathbf{F}_{m}(\xi, \eta), \boldsymbol{\Theta}_{m}(\xi, \eta)\right]\right|,
\end{gathered}
$$

where $\bar{N}_{F}, \bar{N}_{G}$ and $\bar{N}_{\Theta}$ are the governing nonlinear PDEs and $\mathbf{F}_{m}(\xi, \eta)$, $\boldsymbol{\Theta}_{m}(\xi, \eta)$ and $\boldsymbol{\Phi}_{m}(\xi, \eta)$ are the LSPM approximate solutions.

Table 1 displays the approximate numerical solutions of the local skin-friction coefficient, $C_{f x} G r_{x}^{-3 / 4}$ and the local Nusselt number, $N u_{x} G r_{x}^{-1 / 4}$ at different values of the transpiration parameter $\xi$ for Prandtl number $\operatorname{Pr}=0.7$, Hall parameter $m=100$ and magnetic parameter $M=0.5$. The table further shows a comparison of the (LSPM), and the published work of Saha et al. (2007). From the table, it can be seen that the (LSPM) results match perfectly well with those of Saha et al. (2007) up to four decimal digits. In addition, it can be observed from the table that the skin-friction coefficient decreases with an increase in the values of the transpiration parameter while the local Nusselt number increases with an increase in the values of the transpiration parameter. Computed numerical values of the local skin-friction coefficient, $C_{f x} G r_{x}^{1 / 4}$ and the local Nusselt number, $N u_{x} G r_{x}^{1 / 4}$ for different values of the suction parameter $\xi$, when the Prandtl number $\operatorname{Pr}=0.10$ and temperature gradient $n=0.5$ is displayed in Table 2. It can be seen from the Table that there is an excellent agreement between our numerical results and the published result of Hossain et al. Hossain and Paul (2001a). Also, from Table 2, we observe that the values of the local skin-friction coefficient decreases with an increase in the values of the suction parameter while it is noticed that an increase in the values of the suction parameter causes an increase in the values of the local Nusselt number.

Tables 3 depicts a comparison between our numerical values of the local skin-friction $C_{f x} G r_{x}^{1 / 5} / 2$ and the local Nusselt number, $N u_{x} G r_{x}^{1 / 5}$ and the published work of Hossain and Paul (2001b) for different values 
Table 1 Comparison of LSPM and BSQLM approximate solutions for $F^{\prime \prime}(0, \xi)$, and $-\Theta^{\prime}(0, \xi)$, against those of Ref. Saha et al. (2007) at different values of $\xi$ for Equations ( 1 - 3) when $m=100, \operatorname{Pr}=0.7$, and $M=0.5$

\begin{tabular}{|c|ccc|ccc|}
\hline & \multicolumn{3}{|c|}{$F^{\prime \prime}(\xi, 0)$} & \multicolumn{2}{c|}{$-\Theta^{\prime}(\xi, 0)$} \\
\hline$\xi$ & LSPM & BSQLM & $\begin{array}{c}\text { Ref. } \\
\text { Saha et al. (2007) }\end{array}$ & LSPM & BSQLM & $\begin{array}{c}\text { Ref. } \\
\text { Saha et al. (2007) }\end{array}$ \\
\hline 2.5 & 0.5714 & 0.5714 & 0.5717 & 1.7500 & 1.7500 & 1.7499 \\
5 & 0.2857 & 0.2857 & 0.2857 & 3.5000 & 3.5000 & 3.5000 \\
20 & 0.0714 & 0.0714 & 0.0714 & 14.0000 & 14.0000 & 14.0000 \\
40 & 0.0357 & 0.0357 & 0.0357 & 28.0000 & 28.0000 & 28.0000 \\
50 & 0.0286 & 0.0286 & 0.0286 & 35.0000 & 35.0000 & 35.0000 \\
60 & 0.0238 & 0.0238 & 0.0238 & 42.0000 & 42.0000 & 42.0000 \\
70 & 0.0204 & 0.0204 & 0.0204 & 49.0000 & 49.0000 & 49.0000 \\
80 & 0.0179 & 0.0179 & 0.0179 & 56.0000 & 56.0000 & 56.0000 \\
\hline CPU time (sec) & 0.001988 & 80.508407 & & 0.001988 & 80.508407 & \\
\hline
\end{tabular}

Table 2 Comparison of LSPM and BSQLM numerical values of skin friction $\left(F^{\prime \prime}(\xi, 0)\right)$ and Nusselt number $\left(-\Theta^{\prime}(\xi, 0)\right)$ solutions against those of Ref. Hossain and Paul (2001a) at different values of $\xi$ for Equations (6 - 7) when $n=0.5$, and $\operatorname{Pr}=0.10$

\begin{tabular}{|c|c|c|c|c|c|c|}
\hline \multirow[b]{2}{*}{$\xi$} & \multicolumn{3}{|c|}{$F^{\prime \prime}(\xi, 0)$} & \multicolumn{3}{|c|}{$-\Theta^{\prime}(\xi, 0)$} \\
\hline & LSPM & BSQLM & $\begin{array}{c}\text { Ref. } \\
\text { Hossain and Paul (2001a) }\end{array}$ & LSPM & BSQLM & $\begin{array}{c}\text { Ref. } \\
\text { Hossain and Paul (2001a) }\end{array}$ \\
\hline 15 & 0.66387 & 0.66385 & 0.66378 & 1.50269 & 1.50268 & 1.49941 \\
\hline 20 & 0.49933 & 0.49932 & 0.49932 & 2.00114 & 2.00113 & 1.99975 \\
\hline 25 & 0.39978 & 0.39978 & 0.39978 & 2.50058 & 2.50058 & 2.49987 \\
\hline 30 & 0.33324 & 0.33324 & 0.33324 & 3.00034 & 3.00034 & 2.99993 \\
\hline CPU time (sec) & 0.006431 & 79.238710 & & 0.0 .006431 & 79.238710 & \\
\hline
\end{tabular}

Table 3 Comparison of LSPM and BSQLM numerical values of skin friction $\left(F^{\prime \prime}(\xi, 0)\right)$ and Nusselt number $\left(\frac{\xi}{\Phi(\xi, 0)}\right)$ solutions against those of Ref. Hossain and Paul (2001b) at different values of $\xi$ for Equations $(10-11)$ when $m=0.5$, and $\operatorname{Pr}=0.10$

\begin{tabular}{|c|ccc|ccc|}
\hline & \multicolumn{3}{|c|}{$F^{\prime \prime}(\xi, 0)$} & & $\left(\frac{\xi}{\Phi(\xi, 0)}\right)$ \\
\hline$\xi$ & LSPM & BSQLM & $\begin{array}{c}\text { Ref. } \\
\text { and Paul (2001b) }\end{array}$ & LSPM & BSQLM & $\begin{array}{c}\text { Ref. } \\
\text { Hossain and Paul (2001b) }\end{array}$ \\
& & & Hossain & & 1.00950 \\
20 & 0.97055 & 0.97073 & 0.98963 & 1.00884 & 0.004 & 2.00059 \\
30 & 0.24981 & 0.24976 & 0.24953 & 2.00057 & 2.00057 & 3.00012 \\
40 & 0.11110 & 0.11108 & 0.11108 & 3.00011 & 3.00011 & 4.00004 \\
50 & 0.06250 & 0.06250 & 0.06250 & 4.00004 & 4.00004 & 5.00002 \\
\hline CPU time (sec) & 0.04000 & 0.04000 & 0.04000 & 5.00002 & 5.00002 & \\
\hline
\end{tabular}

of the suction parameter $\xi$, when Prandtl number $\operatorname{Pr}=0.10$ and the heat flux gradient $m=0.5$. On comparison, we observe that there is a good agreement between the (LSPM), and the approximate numerical solutions obtained by Hossain and Paul (2001b). We observe from the Table 4 that for increased values of $\xi$, there is a decrease in the values of the local skin-friction, while it is noticed that as $\xi$ increases, the local Nusselt number increases. We remark that the (LSPM) is computationally faster than the (BSQLM) in terms of computational time as accurate solutions are obtained in a fraction of seconds in all the examples considered in this investigation.

Tables 4, 5 and 8 illustrates the results for the skin-friction and the Nusselt number respectively. The tables give a comparison between the (LSPM) and the (BSQLM) numerical approximate solutions and the two results are in good agreement for all values of $\xi$ considered. Again it can be seen from the tables that the (LSPM) gives results in a fraction of a second when compared with the (BSQLM). This is because, in the (LSPM), discretization is done only in the $\xi$-direction while discretization is done both in the $\eta-$ and $\xi$ - direction in the (BSQLM). In particular, it can be observed from the tables that only a few terms of the (LSPM) approximation are required to give results presented in the tables for all large values of $\xi$ considered. This is a clear indication that the (LSPM) is a good numerical tool for solving nonlinear PDEs involving large parameter.

Tables 7 - 9 shows the (LSPM) maximum errors between the current and previous iteration level. The errors norm were used to measure the convergence of the solution algorithm over a number of iterations. It can be seen from the tables that even as $\xi$ becomes very large, the accuracy of the method improves. We also note from the tables that the solution error decreases with an increase in the order of LSPM approximation $K$. The solution error improves as $\xi$ becomes larger. This is evident from the convergence level in table 7 with Error $F, E_{F}$ which is $10^{-16}$ for $\xi=5$, $10^{-23}$ for $\xi=10$ and $10^{-30}$ for $\xi=20$, Error $\Theta, E_{\Theta}$ up to $10^{-15}$ for $\xi=5,10^{-21}$ for $\xi=10$ and $10^{-27}$ for $\xi=20$. In table 8 , accurate results with Error $F, E_{F}$ of order up to $10^{-26}$ for $\xi=5,10^{-42}$ for $\xi=10$ and $10^{-58}$ for $\xi=20$, Error $\Theta, E_{\Theta}$ up to $10^{-26}$ for $\xi=5,10^{-23}$ for $\xi=10$ and $10^{-30}$ for $\xi=20$. Also, in table 9, accurate results with Error $F, E_{F}$ of order up to $10^{-22}$ for $\xi=5,10^{-34}$ for $\xi=10$ and $10^{-47}$ for $\xi=20$, Error $\Phi, E_{\Phi}$ up to $10^{-21}$ for $\xi=5,10^{-33}$ for $\xi=10$ and $10^{-43}$ for $\xi=20$. We note that the results presented in Tables ( 7 - 9) were achieved after nine approximations only. We remark that even at very large values of $\xi$, the LSPM gives accurate results with 
Table 4 Comparison between the LSPM and BSQLM numerical values of the skin friction $F^{\prime \prime}(0, \xi)$ and the Nusselt number $\Theta^{\prime}(0, \xi)$ at different values of $\xi$ for Equations ( 1 - 3) when $m=100, M=0.5$, and $\operatorname{Pr}=0.7$

\begin{tabular}{|c|ccc|ccc|}
\hline$\xi$ & \multicolumn{3}{|c|}{$F^{\prime \prime}(\xi, 0)$} & \multicolumn{2}{c|}{$-\Theta^{\prime}(\xi, 0)$} \\
\hline & $K$ & LSPM & BSQLM & $K$ & LSPM & BSQLM \\
\hline 2 & 10 & 0.714266 & 0.714691 & 8 & 1.400001 & 1.400096 \\
5 & 3 & 0.285713 & 0.285713 & 2 & 3.500000 & 3.500000 \\
10 & 2 & 0.142857 & 0.142857 & 2 & 7.000000 & 7.000000 \\
15 & 1 & 0.095238 & 0.095238 & 1 & 10.500000 & 10.500000 \\
20 & 1 & 0.071429 & 0.071429 & 1 & 14.000000 & 14.000000 \\
30 & 1 & 0.047619 & 0.047619 & 1 & 21.000000 & 21.000000 \\
40 & 1 & 0.035714 & 0.035714 & 1 & 28.000000 & 28.000000 \\
50 & 1 & 0.028571 & 0.028571 & 1 & 35.000000 & 35.000000 \\
\hline CPU time (sec) & & 0.009417 & 80.238359 & & 0.009417 & 80.238359 \\
\hline
\end{tabular}

Table 5 Comparison between the LSPM and BSQLM numerical values of the skin friction $F^{\prime \prime}(0, \xi)$ and the Nusselt number $\Theta^{\prime}(0, \xi)$ at different values of $\xi$ for Equations (6 - 7) when $n=0.5$, and $\operatorname{Pr}=0.7$

\begin{tabular}{|c|ccc|ccc|}
\hline$\xi$ & \multicolumn{3}{|c|}{$F^{\prime \prime}(\xi, 0)$} & \multicolumn{2}{c|}{$-\Theta^{\prime}(\xi, 0)$} \\
\hline & $K$ & LSPM & BSQLM & $K$ & LSPM & BSQLM \\
\hline 2 & 16 & 0.618112 & 0.618530 & 15 & 1.481166 & 1.481091 \\
5 & 3 & 0.284272 & 0.284256 & 3 & 3.506668 & 3.506668 \\
10 & 3 & 0.142812 & 0.142812 & 3 & 7.000840 & 7.000840 \\
15 & 2 & 0.095232 & 0.095232 & 2 & 10.500249 & 10.500249 \\
20 & 2 & 0.071427 & 0.071427 & 2 & 14.000105 & 14.000105 \\
30 & 1 & 0.047619 & 0.047619 & 2 & 21.000031 & 21.000031 \\
40 & 1 & 0.035714 & 0.035714 & 2 & 28.000013 & 28.000013 \\
50 & 1 & 0.028571 & 0.028571 & 2 & 35.000007 & 35.000007 \\
\hline CPU time $(\mathrm{sec})$ & & 0.009417 & 80.238359 & & 0.009417 & 80.238359 \\
\hline
\end{tabular}

Table 6 Comparison between the LSPM and BSQLM numerical values of the skin friction $F^{\prime \prime}(0, \xi)$ and the Nusselt number $\left(\frac{\xi}{\Phi(\xi, 0)}\right)$ at different values of $\xi$ for Equations (10 - 11) when $m=0.5$, and $\operatorname{Pr}=0.7$

\begin{tabular}{|c|ccc|ccc|}
\hline$\xi$ & \multicolumn{3}{|c|}{$F^{\prime \prime}(\xi, 0)$} & & $\left(\frac{\xi}{\Phi(\xi, 0)}\right)$ \\
\hline & $K$ & LSPM & BSQLM & $K$ & LSPM & BSQLM \\
\hline 2 & 13 & 0.438549 & 0.438527 & 15 & 1.460329 & 1.460331 \\
5 & 3 & 0.081469 & 0.081469 & 3 & 3.501916 & 3.501916 \\
10 & 2 & 0.020407 & 0.020407 & 2 & 7.000120 & 7.000120 \\
15 & 1 & 0.009070 & 0.009070 & 2 & 10.500024 & 10.500024 \\
20 & 1 & 0.005102 & 0.005102 & 2 & 14.000008 & 14.000008 \\
30 & 1 & 0.002268 & 0.002268 & 2 & 21.000001 & 21.000001 \\
40 & 1 & 0.001276 & 0.001276 & 1 & 28.000000 & 28.000000 \\
50 & 1 & 0.000816 & 0.000816 & 1 & 35.000000 & 35.000000 \\
\hline CPU time (sec) & & 0.005897 & 78.838219 & & 0.005897 & 78.838219 \\
\hline
\end{tabular}


Table 7 LSPM Convergence of solution maximum error for Equations (1 - 3) at different values of $\xi$ when $m=100, P r=0.7, M=0.5, L=30$, and $N_{x}=60$

\begin{tabular}{|c|c|c|c|c|}
\hline$\xi$ & $K$ & $\mid$ Error $F \|_{\infty}$ & $K$ & $\mid$ Error $\Theta \|_{\infty}$ \\
\hline \multirow[t]{9}{*}{5} & 1 & $4.503 \times 10^{-9}$ & 1 & $1.052 \times 10^{-8}$ \\
\hline & 2 & $3.065 \times 10^{-10}$ & 2 & $1.218 \times 10^{-9}$ \\
\hline & 3 & $3.552 \times 10^{-11}$ & 3 & $1.279 \times 10^{-10}$ \\
\hline & 4 & $3.498 \times 10^{-12}$ & 4 & $1.478 \times 10^{-11}$ \\
\hline & 5 & $5.436 \times 10^{-13}$ & 5 & $2.260 \times 10^{-12}$ \\
\hline & 6 & $6.645 \times 10^{-14}$ & 6 & $2.818 \times 10^{-13}$ \\
\hline & 7 & $1.241 \times 10^{-14}$ & 7 & $5.249 \times 10^{-14}$ \\
\hline & 8 & $1.767 \times 10^{-15}$ & 8 & $7.493 \times 10^{-15}$ \\
\hline & 9 & $3.771 \times 10^{-16}$ & 9 & $1.599 \times 10^{-15}$ \\
\hline \multirow[t]{9}{*}{10} & 1 & $3.518 \times 10^{-11}$ & 1 & $2.478 \times 10^{-10}$ \\
\hline & 2 & $5.985 \times 10^{-13}$ & 2 & $1.904 \times 10^{-11}$ \\
\hline & 3 & $1.734 \times 10^{-14}$ & 3 & $4.996 \times 10^{-13}$ \\
\hline & 4 & $4.270 \times 10^{-16}$ & 4 & $1.443 \times 10^{-14}$ \\
\hline & 5 & $1.659 \times 10^{-17}$ & 5 & $5.517 \times 10^{-16}$ \\
\hline & 6 & $1.720 \times 10^{-19}$ & 6 & $4.719 \times 10^{-17}$ \\
\hline & 7 & $2.366 \times 10^{-20}$ & 7 & $8.009 \times 10^{-19}$ \\
\hline & 8 & $8.425 \times 10^{-22}$ & 8 & $2.859 \times 10^{-20}$ \\
\hline & 9 & $4.496 \times 10^{-23}$ & 9 & $1.525 \times 10^{-21}$ \\
\hline \multirow[t]{9}{*}{20} & 1 & $2.749 \times 10^{-13}$ & 1 & $2.743 \times 10^{-12}$ \\
\hline & 2 & $1.169 \times 10^{-15}$ & 2 & $2.974 \times 10^{-13}$ \\
\hline & 3 & $8.469 \times 10^{-18}$ & 3 & $1.951 \times 10^{-15}$ \\
\hline & 4 & $5.213 \times 10^{-20}$ & 4 & $1.409 \times 10^{-17}$ \\
\hline & 5 & $5.063 \times 10^{-22}$ & 5 & $1.347 \times 10^{-19}$ \\
\hline & 6 & $3.868 \times 10^{-24}$ & 6 & $1.050 \times 10^{-21}$ \\
\hline & 7 & $4.514 \times 10^{-26}$ & 7 & $1.222 \times 10^{-23}$ \\
\hline & 8 & $4.017 \times 10^{-28}$ & 8 & $1.090 \times 10^{-25}$ \\
\hline & 9 & $5.359 \times 10^{-30}$ & 9 & $1.454 \times 10^{-27}$ \\
\hline
\end{tabular}

Table 8 LSPM Convergence of solution maximum error for Equations (6 - 7) at different values of $\xi$ when $n=0.5, \operatorname{Pr}=0.7, L=30$, and $N_{x}=60$

\begin{tabular}{|c|cccc|}
\hline$\xi$ & $K$ & $\|$ Error F $\|_{\infty}$ & $K$ & $\|$ Error $\Theta \|_{\infty}$ \\
\hline 5 & 1 & $4.776 \times 10^{-7}$ & 1 & $2.498 \times 10^{-6}$ \\
& 2 & $2.337 \times 10^{-9}$ & 2 & $8.667 \times 10^{-9}$ \\
& 3 & $9.531 \times 10^{-12}$ & 3 & $2.151 \times 10^{-11}$ \\
& 4 & $2.744 \times 10^{-14}$ & 4 & $2.881 \times 10^{-14}$ \\
& 5 & $2.772 \times 10^{-17}$ & 5 & $2.811 \times 10^{-16}$ \\
& 6 & $3.134 \times 10^{-19}$ & 6 & $9.070 \times 10^{-19}$ \\
& 7 & $1.372 \times 10^{-21}$ & 7 & $2.424 \times 10^{-21}$ \\
& 8 & $2.435 \times 10^{-24}$ & 8 & $2.703 \times 10^{-23}$ \\
& 9 & $1.457 \times 10^{-26}$ & 9 & $7.380 \times 10^{-26}$ \\
\hline 10 & 1 & $5.830 \times 10^{-11}$ & 1 & $2.440 \times 10^{-9}$ \\
& 2 & $8.916 \times 10^{-15}$ & 2 & $2.645 \times 10^{-13}$ \\
& 3 & $1.136 \times 10^{-18}$ & 3 & $2.051 \times 10^{-17}$ \\
& 4 & $1.022 \times 10^{-22}$ & 4 & $8.586 \times 10^{-22}$ \\
& 5 & $3.227 \times 10^{-27}$ & 5 & $2.618 \times 10^{-25}$ \\
& 6 & $1.140 \times 10^{-30}$ & 6 & $2.640 \times 10^{-29}$ \\
& 7 & $1.559 \times 10^{-34}$ & 7 & $2.205 \times 10^{-33}$ \\
& 8 & $8.652 \times 10^{-39}$ & 8 & $7.681 \times 10^{-37}$ \\
& 9 & $1.617 \times 10^{-42}$ & 9 & $6.554 \times 10^{-41}$ \\
\hline 20 & 1 & $7.116 \times 10^{-15}$ & 1 & $2.382 \times 10^{-12}$ \\
& 2 & $3.401 \times 10^{-20}$ & 2 & $8.072 \times 10^{-18}$ \\
& 3 & $1.354 \times 10^{-25}$ & 3 & $1.956 \times 10^{-23}$ \\
& 4 & $3.808 \times 10^{-31}$ & 4 & $2.559 \times 10^{-29}$ \\
& 5 & $3.757 \times 10^{-37}$ & 5 & $2.439 \times 10^{-34}$ \\
& 6 & $4.147 \times 10^{-42}$ & 6 & $7.683 \times 10^{-40}$ \\
& 7 & $1.773 \times 10^{-47}$ & 7 & $7.122 \times 10^{-37}$ \\
& 8 & $3.474 \times 10^{-53}$ & 8 & $2.183 \times 10^{-50}$ \\
& 9 & $1.795 \times 10^{-58}$ & 9 & $5.821 \times 10^{-56}$ \\
\hline
\end{tabular}


Table 9 LSPM Convergence of solution maximum error for Equations $(10-11)$ at different values of $\xi$ when $m=0.5, P r=0.7, L=40$, and $N_{x}=60$

\begin{tabular}{|c|c|c|c|c|}
\hline$\xi$ & $K$ & $\mid$ Error $F \|_{\infty}$ & $K$ & $\mid$ Error $\Phi \|_{\infty}$ \\
\hline \multirow[t]{9}{*}{5} & 1 & $3.145 \times 10^{-6}$ & 1 & $1.007 \times 10^{-5}$ \\
\hline & 2 & $4.874 \times 10^{-8}$ & 2 & $1.094 \times 10^{-7}$ \\
\hline & 3 & $6.410 \times 10^{-10}$ & 3 & $9.282 \times 10^{-10}$ \\
\hline & 4 & $6.182 \times 10^{-12}$ & 4 & $5.598 \times 10^{-12}$ \\
\hline & 5 & $2.671 \times 10^{-14}$ & 5 & $1.038 \times 10^{-13}$ \\
\hline & 6 & $6.570 \times 10^{-16}$ & 6 & $1.267 \times 10^{-15}$ \\
\hline & 7 & $1.019 \times 10^{-17}$ & 7 & $1.314 \times 10^{-17}$ \\
\hline & 8 & $7.697 \times 10^{-20}$ & 8 & $4.187 \times 10^{-19}$ \\
\hline & 9 & $9.140 \times 10^{-22}$ & 9 & $4.600 \times 10^{-21}$ \\
\hline \multirow[t]{9}{*}{10} & 1 & $1.536 \times 10^{-9}$ & 1 & $3.935 \times 10^{-8}$ \\
\hline & 2 & $1.487 \times 10^{-12}$ & 2 & $2.671 \times 10^{-11}$ \\
\hline & 3 & $1.223 \times 10^{-15}$ & 3 & $1.416 \times 10^{-14}$ \\
\hline & 4 & $7.370 \times 10^{-19}$ & 4 & $5.399 \times 10^{-18}$ \\
\hline & 5 & $1.990 \times 10^{-22}$ & 5 & $6.189 \times 10^{-21}$ \\
\hline & 6 & $3.060 \times 10^{-25}$ & 6 & $4.719 \times 10^{-24}$ \\
\hline & 7 & $2.965 \times 10^{-28}$ & 7 & $3.059 \times 10^{-27}$ \\
\hline & 8 & $1.400 \times 10^{-31}$ & 8 & $6.092 \times 10^{-30}$ \\
\hline & 9 & $1.039 \times 10^{-34}$ & 9 & $4.183 \times 10^{-33}$ \\
\hline \multirow[t]{9}{*}{20} & 1 & $7.499 \times 10^{-13}$ & 1 & $1.537 \times 10^{-10}$ \\
\hline & 2 & $4.539 \times 10^{-17}$ & 2 & $6.520 \times 10^{-15}$ \\
\hline & 3 & $2.332 \times 10^{-21}$ & 3 & $2.161 \times 10^{-19}$ \\
\hline & 4 & $8.785 \times 10^{-26}$ & 4 & $5.092 \times 10^{-24}$ \\
\hline & 5 & $1.483 \times 10^{-30}$ & 5 & $3.689 \times 10^{-28}$ \\
\hline & 6 & $1.425 \times 10^{-34}$ & 6 & $1.758 \times 10^{-32}$ \\
\hline & 7 & $8.629 \times 10^{-39}$ & 7 & $7.122 \times 10^{-37}$ \\
\hline & 8 & $2.547 \times 10^{-43}$ & 8 & $8.865 \times 10^{-41}$ \\
\hline & 9 & $1.181 \times 10^{-47}$ & 9 & $3.805 \times 10^{-45}$ \\
\hline
\end{tabular}

a very small solution error. This is one of the most interesting finding of this investigation. This further indicates that the (LSPM) is a suitable numerical method for solving nonlinear PDEs similar to those considered in this work.

Tables 10 - 12 displays the (LSPM) residual error for $F, \Theta$ and $\Phi$ respectively. It can be seen from the tables that the saturation level is at least $10^{-9}$ in the equation for $F(\eta, \xi), 10^{-12}$ in the equation $\Theta(\eta, \xi)$ and at $10^{-12}$ in the equation $\Phi(\eta, \xi)$. This shows that even when $\xi$ is very large, very accurate results can be obtained which is in contrast with the existing (SPM) known in the literature.

\section{CONCLUSIONS}

In this paper, we have discussed the application of the large parameter spectral perturbation method (LSPM) on systems of nonlinear PDEs. The large parameter spectral perturbation method (LSPM) is used to solve the equations describing the effect of hall current on the MHD laminar natural convection flow from a vertical permeable flat with uniform surface temperature, free convection from a vertical permeable circular cone with a non-uniform surface, and free convection from a vertical permeable circular cone with non-uniform surface heat flux previously investigated by Saha et al. (2007), Hossain and Paul (2001a) and Hossain and Paul (2001b), respectively. The purpose of the present study is to present a compliment of the existing spectral perturbation method that solves fluid mechanics problems with large parameters. Also, we have been able to show that the range of validity of the standard spectral perturbation method (SPM) can be extended by expanding about a large physical parameter so as to make the standard (SPM) robust, efficient and extend its application to new areas. From the numerical simulations, some conclusions can be drawn as follows;
- The results become more accurate even as $\xi$ becomes larger. This observation contradicts the standard (SPM) which does not give accurate results as $\xi$ approaches 1 . We remark also that very few terms of the (LSPM) is required to obtain converged results presented in the Tables.

- Significantly few seconds was required to attain desired converged results that are comparable with published literature. The computational speed of our approach is primarily due to the fact that with the spectral collocation method, only few grid points are required to yield accurate results. Hence, it is concluded from the observations made that the (LSPM) is computationally fast.

- The (LSPM) can be used as an alternative numerical approach to get numerical solutions for higher order asymptotic series equations that are not possible to find, or very difficult to find with the usual asymptotic perturbation schemes.

- The ease of implementation, computational speed and accuracy of the (LSPM) suggest that this method can be used to extend the range of validity of the standard (SPM), and improves the convergence rate of the usual (SPM) even as $\xi$ becomes larger in as much the series expansion is about a large parameter.

For problems related to those investigated in this paper, the (LSPM) can be used as an alternative numerical tool for obtaining numerical approximate solutions. 
Table 10 LSPM residual error for Equations (1 - 3) at different values of $\xi$ when $m=100, \operatorname{Pr}=0.7, M=0.5, L=40$, and $N_{x}=60$

\begin{tabular}{|c|cc|}
\hline$\xi$ & $\|$ Residual Error $F \|_{\infty}$ & $\|$ Residual Error $\Phi \|_{\infty}$ \\
\hline 2 & $2.850 \times 10^{-4}$ & $3.865 \times 10^{-3}$ \\
5 & $2.747 \times 10^{-9}$ & $6.110 \times 10^{-11}$ \\
10 & $3.212 \times 10^{-9}$ & $1.472 \times 10^{-12}$ \\
15 & $2.640 \times 10^{-9}$ & $6.168 \times 10^{-12}$ \\
20 & $2.805 \times 10^{-9}$ & $3.240 \times 10^{-12}$ \\
30 & $2.922 \times 10^{-9}$ & $4.349 \times 10^{-12}$ \\
40 & $2.640 \times 10^{-9}$ & $2.899 \times 10^{-12}$ \\
50 & $3.237 \times 10^{-9}$ & $1.727 \times 10^{-12}$ \\
\hline
\end{tabular}

Table 11 LSPM residual error for Equations (6 - 7) at different values of $\xi$ when $n=0.5, \operatorname{Pr}=0.7, L=40$, and $N_{x}=60$

\begin{tabular}{|c|cc|}
\hline$\xi$ & $\|$ Residual Error $F \|_{\infty}$ & $\|$ Residual Error $\Phi \|_{\infty}$ \\
\hline 2 & $9.391 \times 10^{-3}$ & $9.536 \times 10^{-3}$ \\
5 & $2.915 \times 10^{-9}$ & $3.448 \times 10^{-6}$ \\
10 & $3.227 \times 10^{-9}$ & $1.355 \times 10^{-8}$ \\
15 & $2.363 \times 10^{-9}$ & $5.287 \times 10^{-10}$ \\
20 & $3.881 \times 10^{-9}$ & $5.291 \times 10^{-11}$ \\
30 & $3.261 \times 10^{-9}$ & $3.581 \times 10^{-12}$ \\
40 & $2.956 \times 10^{-9}$ & $2.685 \times 10^{-12}$ \\
50 & $2.952 \times 10^{-9}$ & $4.206 \times 10^{-12}$ \\
\hline
\end{tabular}

Table 12 LSPM residual error for Equations $(10-11)$ at different values of $\xi$ when $m=0.5, P r=0.7, L=40$, and $N_{x}=60$

\begin{tabular}{|c|cc|}
\hline$\xi$ & $\|$ Residual Error F $\|_{\infty}$ & $\|$ Residual Error $\Phi \|_{\infty}$ \\
\hline 2 & $3.619 \times 10^{-5}$ & $3.950 \times 10^{-3}$ \\
5 & $5.434 \times 10^{-9}$ & $5.069 \times 10^{-7}$ \\
10 & $3.571 \times 10^{-9}$ & $4.960 \times 10^{-10}$ \\
15 & $3.797 \times 10^{-9}$ & $8.561 \times 10^{-12}$ \\
20 & $4.415 \times 10^{-9}$ & $2.430 \times 10^{-12}$ \\
30 & $5.113 \times 10^{-9}$ & $2.449 \times 10^{-12}$ \\
40 & $4.021 \times 10^{-9}$ & $7.191 \times 10^{-12}$ \\
50 & $5.054 \times 10^{-9}$ & $2.216 \times 10^{-12}$ \\
\hline
\end{tabular}

\section{ACKNOWLEDGMENT}

The authors wish to thank the University of KwaZulu-Natal and DSTNRF Centre of Excellence in Mathematical and Statistical Sciences (CoEMaSS), South Africa for necessary support.

\section{REFERENCES}

Agbaje, T.M., Motsa, S.S., 2015 "Comparison between Spectral Perturbation and Spectral Relaxation Approach for Unsteady Heat and Mass Transfer by MHD Mixed Convection Flow over an Impulsively Stretched Vertical Surface with Chemical Reaction Effect," Journal of Interpolation and Approximation in Scientific Computing 2015, (1), 48-83. http://doi:10.5899/2015/jiasc-00076

Bhattacharyya, K., 2013, "Boundary Layer Stagnation-Point Flow of Casson Fluid and Heat Transfer Towards a Shrinking/Stretching Sheet," Frontiers in Heat and Mass Transfer 4, 1-9, Article ID 023003. http: //dx.doi.org/10.5098/hmt.v4.2.3003

Chamkha, A.J., Takhar, H.S., Nath, G., 2003, "Unsteady MHD Rotating Flow over a Rotating Sphere near the Equator," Acta Mechanica 164, (12), 31-46.

https://doi.org/10.1007/s00707-003-0011-z

Canuto, C., Hussaini, M.Y., Quarteroni, A., Zang, T.A., 1988, "Spectral Methods in Fluid Dynamics," Springer-Verlag, Berlin.

https://doi.org/10.1007/978-3-642-84108-8
Gorla, R.S.R., Slaouti, A., Takhar, H.S., 1998, "Free Convection in Micropolar Fluids over a Uniformly Heated Vertical Plate," International Journal Numerical Methods for Heat \& Fluid Flow 8, (5), 504-518. https://doi.org/10.1108/09615539810220261

Hossain, M.A., Paul, S.C., 2001, "Free Convection from a Vertical Permeable Circular Cone with Non-uniform Surface Temperature," Acta Mechanica 151, (1-2), 103-114. https://doi.org/10.1007/BF01272528

Hossain, M.A., Paul, S.C., 2001, "Free Convection from a Vertical Permeable Circular Cone with Non-uniform Surface Heat Flux," Heat and Mass Transfer 37, (2-3), 167-173.

https://doi.org/10.1007/s002310000129

Hussain, S., Hossain, M.A., Wilson, M., 2000, "Natural Convection Flow from a Vertical Permeable Flat Plate with Variable Surface Temperature and Species Concentration," Engineering Computations 17, (7), 789812.

https://doi.org/10.1108/02644400010352261

Motsa, S.S., Magagula, V.M., Sibanda, P., 2014, "A Bivariate Chebyshev Spectral Collocation Quasilinearization Method for Nonlinear Evolution Parabolic Equations," The Scientific World Journal 2014, Article ID 581987, 13 pages.

http://dx.doi.org/10.1155/2014/581987

Motsa, S.S., Ansari, M.S., 2015, "Unsteady Boundary Layer Flow and Heat Transfer of Oldroyd-B Nanofluid towards a Stretching Sheet with 
Variable Thermal Conductivity," Thermal Science 19, (1), 239-248. https://doi.org/10.2298/TSCI15S1S39M

Oyelakin, I.S., Mondal, S., Sibanda, P., 2017, "Unsteady MHD Three - dimensional Casson Nanofluid Flow over a Porous Linear Stretching Sheet with Slip Condition," Frontiers in Heat and Mass Transfer 8 37, $1-9$.

DOI: $10.5098 / \mathrm{hmt} .8 .37$

Roy, S., Takhar, H.S., Nath, G., 2004, "Unsteady MHD Flow on a Rotating Cone in a Rotating Fluid," Meccanica 39, (3), 271- 283.

https://doi.org/10.1023/B:MECC.0000022847.28148.98

Saha, L.K., Hossain, M.A., Gorla, R.S.R., 2007, "Effect of Hall Current on the MHD Laminar Natural Convection Flow from a Vertical Permeable Flat Plate with Uniform Surface Temperature," International Journal of Thermal Sciences 46, (8), 790-801.

https://doi.org/10.1016/j.ijthermalsci.2006.10.009
Slaouti, A., Takhar, H.S., Nath, G., 2002 "Spin-up and Spin-down of a Viscous Fluid over a Heated Disk Rotating in a Vertical Plane in the Presence of a Magnetic Field and a Buoyancy Force," Acta Mechanica 156, (1-2), 109-129.

https://doi.org/10.1007/BF01188745

Takhar, H.S., Chamkha, A.J., Nath, G., 2001, "Unsteady Threedimensional MHD-boundary-layer Flow due to the Impulsive Motion of a Stretching Surface," Acta Mechanica 146, (1-2), 59-71. https://doi.org/10.1007/BF01178795

Takhar, H.S., Nath, G., Singh, A.K., 2001,"Unsteady MHD Boundarylayer of a Source and Vortex Flow Adjacent to a Stationary Surface," Acta Mechanica 146, (1-2), 9-20. https://doi.org/10.1007/BF01178791

Trefethen, L.N., 2000, "Spectral Methods in MATLAB," SIAM. https://doi.org/10.1137/1.9780898719598.bm 